\title{
Matroids and Integrality Gaps for Hypergraphic Steiner Tree Relaxations
}

\author{
Michel X. Goemans ${ }^{*}$ \\ Massachusetts Institute of \\ Technology \\ Cambridge, USA \\ goemans@math.mit.edu \\ Thomas Rothvoß \\ Massachusetts Institute of \\ Technology \\ Cambridge, USA \\ rothvoss@math.mit.edu
}

\author{
Neil Olver ${ }^{\dagger}$ \\ Massachusetts Institute of \\ Technology \\ Cambridge, USA \\ olver@math.mit.edu \\ Rico Zenklusen ${ }^{\S}$ \\ Massachusetts Institute of \\ Technology \\ Cambridge, USA \\ ricoz@math.mit.edu
}

\begin{abstract}
Until recently, LP relaxations have only played a very limited role in the design of approximation algorithms for the Steiner tree problem. In particular, no (efficiently solvable) Steiner tree relaxation was known to have an integrality gap bounded away from 2 , before Byrka et al. [3] showed an upper bound of $\approx 1.55$ of a hypergraphic LP relaxation and presented a $\ln (4)+\epsilon \approx 1.39$ approximation based on this relaxation. Interestingly, even though their approach is LP based, they do not compare the solution produced against the LP value.

We take a fresh look at hypergraphic LP relaxations for the Steiner tree problem-one that heavily exploits methods and results from the theory of matroids and submodular functions-which leads to stronger integrality gaps, faster algorithms, and a variety of structural insights of independent interest. More precisely, along the lines of the algorithm of Byrka et al. [3], we present a deterministic $\ln (4)+\epsilon$ approximation that compares against the LP value and therefore proves a matching $\ln (4)$ upper bound on the integrality gap of hypergraphic relaxations.

Similarly to [3], we iteratively fix one component and update the LP solution. However, whereas in [3] the LP is solved at every iteration after contracting a component, we show how feasibility

\footnotetext{
${ }^{*}$ Supported by NSF grants CCF-1115849 and CCF-0829878, and by ONR grant N00014-11-1-0053.

${ }^{\dagger}$ Supported by NSF grant CCF-1115849.

${ }^{\ddagger}$ Supported by the Alexander von Humboldt Foundation within the Feodor Lynen program, by ONR grant N00014-11-1-0053 and by NSF grants CCF-0829878 and CCF-1115849.

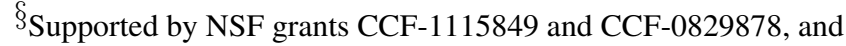
by ONR grants N00014-11-1-0053 and N00014-09-1-0326.
\end{abstract}

Permission to make digital or hard copies of all or part of this work for personal or classroom use is granted without fee provided that copies are not made or distributed for profit or commercial advantage and that copies bear this notice and the full citation on the first page. To copy otherwise, to republish, to post on servers or to redistribute to lists, requires prior specific permission and/or a fee.

STOC'12, May 19-22, 2012, New York, New York, USA.

Copyright 2012 ACM 978-1-4503-1245-5/12/05 ...\$10.00. can be maintained by a greedy procedure on a well-chosen matroid. Apart from avoiding the expensive step of solving a hypergraphic LP at each iteration, our algorithm can be analyzed using a simple potential function. This potential function gives an easy means to determine stronger approximation guarantees and integrality gaps when considering restricted graph topologies. In particular, this readily leads to a $\frac{73}{60} \approx 1.217$ upper bound on the integrality gap of hypergraphic relaxations for quasi-bipartite graphs.

Additionally, for the case of quasi-bipartite graphs, we present a simple algorithm to transform an optimal solution to the bidirected cut relaxation to an optimal solution of the hypergraphic relaxation, leading to a fast $\frac{73}{60}$ approximation for quasi-bipartite graphs. Furthermore, we show how the separation problem of the hypergraphic relaxation can be solved by computing maximum flows, which provides a way to obtain a fast independence oracle for the matroids that we use in our approach.

\section{Categories and Subject Descriptors}

F.2.2 [Computations on discrete structures]: Non-numerical Algorithms and Problems

\section{General Terms}

Algorithms, Theory

\section{Keywords}

Matroids, approximation algorithms, linear programming relaxations, integrality gaps

\section{INTRODUCTION}

The Steiner tree problem is one of the most fundamental and important problems in Computer Science and Operations Research. Whereas a 2-approximation is easily obtained by computing a minimum spanning tree over the terminals, obtaining algorithms with an approximation guarantee bounded away from 2 has proven to be a non-trivial task. The problem is known to be inapproximable to within $\frac{96}{95}$, unless $\mathbf{P}=\mathbf{N P}[1,8]$. There has been a long sequence of combinatorial approximation algorithms [9, 24, 12, 19, 21], based on different greedy approaches, culminating in the famous $1+\frac{\ln (3)}{2}+\epsilon<1.55$ approximation of Robins and Zelikovsky [21]. 
No further progress was achieved until Byrka, Grandoni, Rothvoß and Sanità [3] presented the first LP-based approach leading to a $\ln (4)+\epsilon \approx 1.39$ approximation. Until this work, for no (efficiently solvable) LP relaxation of the Steiner tree problem was it known whether the integrality gap was bounded away from 2 . Intriguingly, even though their $\ln (4)+\epsilon$ approximation algorithm is based on a particular LP relaxation, its approximation guarantee is not with respect to the LP solution and does not imply a $\ln (4)$ integrality gap for the relaxation. In [3], the authors show a weaker $\approx 1.55$ integrality gap using a technique not directly linked to their algorithm. Chakrabarty et al. [7] provide a simpler alternative proof of the same bound.

The linear relaxation used by Byrka et al., the directed componentbased relaxation, was introduced by Polzin and Vahdati-Daneshmand [18], based in turn on an equivalent undirected component-based LP introduced by Warme [23]. It is the undirected version that we will use in this paper. Another notable relaxation is the partition-based LP introduced by Könemann et al. [13]. In [6], Chakrabarty et al. showed that this relaxation is equivalent to the others mentioned above, and introduced the term "hypergraphic" for this family of relaxations. They also proved that basic solutions are sparse, having support size less than the number of terminals.

The limited understanding of LP relaxations of the Steiner tree problem is arguably a major barrier in the design of stronger approximation algorithms. The goal of this work is to fill this gap by providing a fresh view on the component-based LP relaxationone that heavily exploits methods and results from the theory of matroids and submodular functions. More precisely, based on the approach of Byrka et al. [3], we present a deterministic $\ln (4)+\epsilon$ algorithm that starts with a solution to the component-based LP relaxation, iteratively contracts a component and updates the LP solution. The algorithm of Byrka et al. solves the component-based LP (through a very large extended formulation) in each iteration after contracting, in order to again obtain a feasible solution. By contrast, we show how the LP can be modified by a simple greedy algorithm over a well-chosen matroid to achieve the same goal. This leads to a considerably faster way to update the LP, but more importantly, we show how the approximation quality of our approach can be analyzed with respect to the initial LP solution. This implies a bound of the integrality gap of the component-based LP relaxation of $\ln (4)$. By comparison, the best known lower bound is $8 / 7 \approx 1.142$ (e.g., by the example of [13]). Furthermore, we show how the separation problem of the component-based relaxation can be reduced to computing maximum flows. Whereas this result is likely to be of independent interest, it also provides a way to optimize quickly over the matroids that we use in our approach, with a single minimum-cost flow computation.

Additionally, we further investigate the special case of quasibipartite graphs, which has played a central role in the design of approximation algorithms for the Steiner tree problem, as well as to find APX-hard problem classes. Rajagopalan and Vazirani [20] showed that the integrality gap of the bidirected cut relaxation for such graphs can be bounded by $3 / 2$. This was later improved to $4 / 3$ [5] and to 1.28 [7]. We obtain a $\frac{73}{60}$ bound for the integrality gap, again matching the approximation factor of [3]. Such a bound was previously known only for the case when all edge costs are equal [7]. Chakrabarty et al. [6] showed that on quasi-bipartite graphs, the bidirected cut and hypergraphic relaxations are actually equivalent. However their proof is based on a duality argument, and they leave as an open problem the question of converting a solution from the bidirected cut relaxation to the hypergraphic relaxation efficiently (more quickly than simply optimizing the hypergraphic LP). We present a simple algorithm to perform this transformation; since the bidirected cut relaxation can be solved much more efficiently via a compact extended formulation, this gives a much faster method of solving the hypergraphic LP in the quasi-bipartite case. Combining this result with the suggested approximation algorithm, we obtain a significantly faster $\frac{73}{60}$ approximation than the one of Byrka et al. [3], since we do not need to (repeatedly) optimize the componentbased relaxation by using either the ellipsoid method or a very large extended formulation.

Organization. The remainder of the paper is organized as follows. Section 2.1 discusses the hypergraphic LP and some related preliminaries. Sections 2.2 and 2.3 give a high level overview of the analysis and the algorithm. We then take a step deeper into the techniques used in the analysis in Section 3. Section 4 discusses improved results for the special case of quasi-bipartite graphs, as well as the constructive equivalence between the hypergraphic and bidirected cut relaxations.

A number of key technical components can be found in the appendices. Appendix A addresses the question of separation and shows that the underlying matroid is a gammoid. A number of the proofs of results in Section 2 can be found in Appendix B. Appendix C gives the proof of a key technical tool involving a decomposition of intersecting submodular functions. Finally, Appendix D demonstrates that solving the hypergraphic LP exactly is NP-hard.

\section{DISCUSSION OF RESULTS AND TECH- NIQUES}

\subsection{The component-based LP}

Let $G=(V, E)$ be an undirected graph with terminals $R \subseteq V$ and edge costs $c: E \rightarrow \mathbb{R}_{+}$. A component $C$ is simply a subgraph of $G$ with the property that it is a tree spanning $V(C)$, all leaves of $C$ are terminals, and all internal vertices are non-terminals. Write $\operatorname{cost}(C):=\sum_{e \in E(C)} c(e)$ for the cost of a component $C$. We will frequently need the terminal set of a component $C \in \mathcal{K}$, and so by abuse of notation, when we refer to $C$ as a vertex set, we mean the set $V(C) \cap R$ of terminals in $C$. In particular, $|C|$ refers to the number of terminals in $C$.

Now let $\mathcal{K}$ be the set of all components of $G$; we assume that all components contain at least two terminals, else they can be safely removed. We use the notation $(Z)^{+}:=\max \{Z, 0\}$. Then the component-based LP relaxation is as follows [23]:

$$
\begin{array}{rlrl}
\min & \sum_{C \in \mathcal{K}} x_{C} \operatorname{cost}(C) & & \\
\sum_{C \in \mathcal{K}} x_{C}(|S \cap C|-1)^{+} & \leq|S|-1 & & \forall S \subseteq R, S \neq \emptyset \\
\sum_{C \in \mathcal{K}} x_{C}(|C|-1) & =|R|-1 & \\
x_{C} \geq 0 & & \forall C \in \mathcal{K} .
\end{array}
$$

Let us make clear that this is indeed a relaxation for Steiner tree: consider any Steiner tree $T$ and decompose it into a collection $C_{1}, \ldots, C_{q}$ of components. Then letting $x_{C_{i}}:=1$ for all $i=$ $1, \ldots, q$ (and 0 otherwise) defines a feasible solution to (LP). To see this, observe that the left hand side $\sum_{C \in \mathcal{K}} x_{C}(|S \cap C|-1)^{+}$ of the first constraint counts the number of "connections" that the components induce for a subset $S$ of terminals. Since $T$ is cycle-free, this number is bounded by $|S|-1$. The second constraint simply imposes that the total number of "connections" equals $|R|-1$, which is satisfied since $T$ is a tree. 
Borchers and Du [2] showed that the optimal $k$-restricted Steiner tree, meaning only components with at most $k$ terminals can be used, has cost at most $1+1 /\left\lfloor\log _{2} k\right\rfloor$ times the cost of an optimal Steiner tree. Furthermore, when restricting the variables in (LP) to components with at most $k$ terminals, the resulting linear program can be solved efficiently, e.g., by solving a polynomial-size extended formulation [3]. It follows that for any fixed $\epsilon>0$, a $(1+\epsilon)$ approximate solution to (LP) can be obtained efficiently. We also point out in Appendix D that optimizing (LP) exactly is strongly NP-hard (this does not seem to have been previously observed).

The framework of our algorithm is similar to Byrka et al. [4], and in particular, it is iterative in nature. They begin by computing a near-optimal fractional solution $x$ to (LP). They then sample a component $C$ at random, proportional to its entry $x_{C}$, and contract this component. The solution $x$ is no longer feasible to (LP) on this new contracted instance, so they re-solve the LP and iterate this procedure until all terminals are connected.

In their analysis, they show that a single random contraction reduces the cost of the optimum Steiner tree by a certain factor in each iteration. The crucial ingredient is a lower bound on the expected cost of edges that could be removed from an optimum solution after a contraction, while still obtaining a Steiner tree. To obtain a bound on the integrality gap, we need a stronger result that says even a fractional solution becomes significantly cheaper after a random contraction. Even for a fixed set of terminals $Q$, it was unclear how to modify a fractional solution in order to preserve feasibility after contraction - a question that had a simple answer in the integral case. Our first goal will be to obtain an understanding of the structure of these modifications.

While it can be avoided, it significantly simplifies the discussion to consider "blown up" versions of solutions to (LP). Consider any $x \in \mathbb{Q}_{+}^{\mathcal{K}}$, and let $N \in \mathbb{N}$ be such that $x_{C} \cdot N \in \mathbb{N}$ for all $C \in \mathcal{K}$. The minimal blowup graph corresponding to $x$ is the unweighted multigraph $\mathcal{X}$ defined as follows. First take the disjoint union of $x_{C} \cdot N$ disjoint copies of $C$ for each component $C$; then identify, for each $v \in R$, all the copies of $v$. The edge costs of $\mathcal{X}$ are inherited from $G$ in the obvious way. See Figure 1 for an example of an LP solution and its associated minimal blowup graph. Observe then that $\operatorname{cost}(\mathcal{X})=N \cdot \operatorname{cost}(x)$. Note that $\mathcal{X}$ (along with $N$, but this will remain fixed throughout) encodes all the information in $x$. In particular, given $\mathcal{X}$ we can determine all of its components: these are simply the maximal connected subgraphs that are trees whose leaves are precisely the terminals spanned. Thus we can define $\Gamma(\mathcal{X})$ as the set of components of a blowup graph $\mathcal{X}$. Each component $C \in \Gamma(\mathcal{X})$ is a subgraph of $\mathcal{X}$, but again, we will abuse notation when the context is clear and sometimes use $C$ to refer to just the terminals of $C$. Thus, e.g., for some $S \subseteq R, S \cap C$ refers to the terminals in $C$ that are also in $S$.

We will need slightly more generality in our definition of a blowup graph. For any $t \in \mathbb{N}$, let $G_{t}$ be the multigraph obtained by first taking $t$ disjoint copies of $G$, and then for each $v \in R$, identifying all copies of $v$. For a solution $y$ with corresponding minimal blowup graph $\mathcal{Y}$, we call a multigraph $\mathcal{Y}^{\prime}$ a (not necessarily minimal) blowup graph corresponding to $y$ if (i) $\mathcal{Y}^{\prime} \subseteq G_{t}$ for some $t \in \mathbb{N}$, (ii) $\mathcal{Y}^{\prime} \supseteq$ $\mathcal{Y}$, and (iii) for any distinct terminals $u, v \in R$, there is no $u$ - $v$-path in $\mathcal{Y}^{\prime}$ that is not already present in $\mathcal{Y}$. Any edges in $\mathcal{Y}^{\prime}$ that were not in $\mathcal{Y}$ we call pendant edges. We will say that a blowup graph $\mathcal{Y}$ is feasible if it corresponds to a feasible solution to (LP); otherwise we call it infeasible. Note that pendant edges have no effect on feasibility; they will always be removed in what we will later call a "cleanup" step.

\subsection{Edge removal after contraction}

Let $\mathcal{X}$ be the blowup graph corresponding to some solution $x$. We are interested in the situation after contracting some component of $G$. In order to avoid some annoying technicalities, for now instead of contracting $Q$ we will think of increasing the value of $x_{Q}$ by 1. In other words, in terms of the blowup graph, we take $N$ fresh copies of component $Q$ and add it to $\mathcal{X}$. We denote the new blowup graph obtained by $\mathcal{X} \circledast Q$. Formally, $\mathcal{X} \circledast Q$ is obtained by taking the disjoint union of $\mathcal{X}$ and $N$ copies of $Q$, and then identifying all copies of $v$ for each $v \in R$.

It is clear that $\mathcal{X} \circledast Q$ is not feasible. We are interested in describing the set of edges $F \subseteq E(\mathcal{X})$ that can be removed so that $(\mathcal{X} \circledast Q)-F$ is feasible.

This is the primary reason that it is simpler to work with the blowup graph $\mathcal{X}$ rather than $x$; this modification operation is much simpler than an equivalent operation defined on $x$. For example, removing a single edge from $\mathcal{X}$ can have the effect of splitting up some component $C$ into subcomponents $C_{1}$ and $C_{2}$; the corresponding effect on $x$ is to reduce $x_{C}$ by $1 / N$ and increase $x_{C_{1}}$ and $x_{C_{2}}$ by the same amount.

Unfortunately, the set of all possible edge removals is not so well behaved. In order to expose the structure we need, we must consider minimal removals. Let

$$
\mathcal{B}_{Q}=\left\{\begin{array}{l|l}
B \subseteq E(\mathcal{X}) & \begin{array}{l}
(\mathcal{X} \circledast Q)-B \text { is feasible, and } \\
B \text { is minimal with this property }
\end{array}
\end{array}\right\} .
$$

Figure 2 shows an example; after a set $B \in \mathcal{B}_{Q}$ is removed, an edge of the blowup graph becomes pendant, and so can also be removed without affecting feasibility.

One of the most crucial elements of our analysis is the following:

THEOREM 2.1. For every component $Q, \mathcal{B}_{Q}$ forms the set of bases of a matroid $M_{Q}$.

In particular, it follows that any minimal removal set has the same number of edges; this number turns out to be $N(|Q|-1)$. We are able to give a precise description of the matroid $M_{Q}$ by giving its rank function; more details of this will be given in Section 3. We can also show that the matroid is a gammoid (a special type of matroid related to flows [22]); see Appendix A. As an aside, we note that $M_{Q}$ depends only on the terminals of $Q$, and not its structure; we could actually define a matroid $M_{S}$ for any subset $S$ of terminals, but this will not be important for our purposes.

We will now study which edge sets can be removed after the random contraction of a component. Even though we will finally present a deterministic algorithm, this analysis will be helpful in guaranteeing the existence of removal sets with certain properties by an averaging argument.

As before, let $\mathcal{X}$ be the blowup graph corresponding to a feasible LP solution $x$. Upon contracting component $Q$, we may remove some edges in order to again obtain a feasible solution. In particular, by Theorem 2.1, we can remove any basis of $M_{Q}$. For added flexibility, we allow choosing a basis $B_{Q} \in \mathcal{B}_{Q}$ randomly, according to any distribution we like. In this case, each edge $e$ will be removed with some probability $q_{e}$. The probability vectors that are attainable are simply the convex combinations of incidence vectors of the bases; in other words, precisely the vectors in $B\left(M_{Q}\right)$, the base polytope of $M_{Q}$.

Now consider, as in [4], randomly contracting a single component, with component $Q \in \Gamma(\mathcal{X})$ contracted with probability $1 /|\Gamma(\mathcal{X})|$. Note that since each original component $\tilde{Q} \in \mathcal{K}$ has $N x_{\tilde{Q}}$ copies 


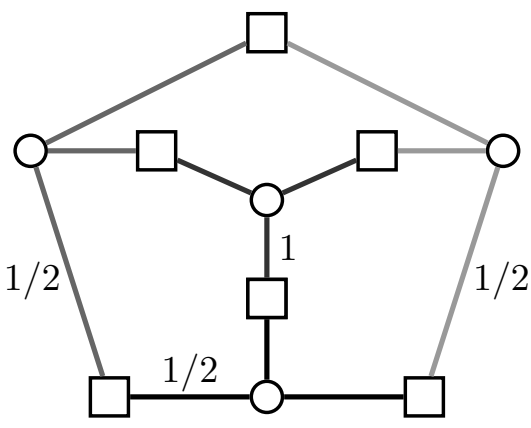

(a) fractional solution $x$

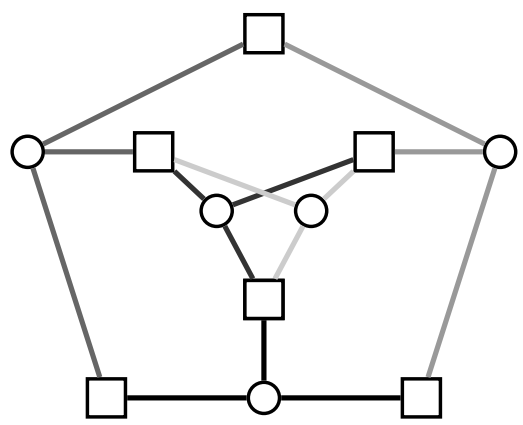

(b) blowup graph $\mathcal{X}$

Figure 1: In $(a)$ : fractional solution $x$ (components drawn in different gray scales and labelled with their capacity $\left.x_{C}\right)$. In $(b)$ : blowup graph $\mathcal{X}$ for $N=2$.

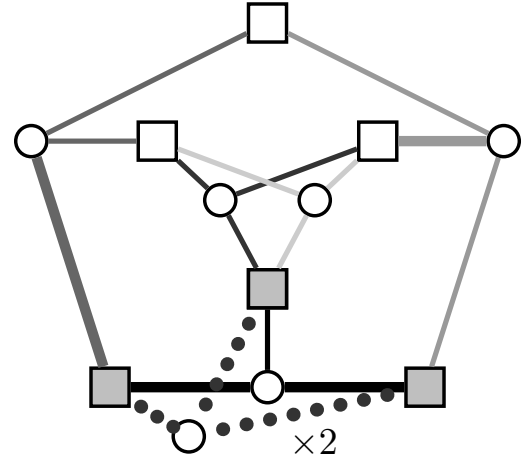

(a) blowup graph $\mathcal{X} \circledast Q$

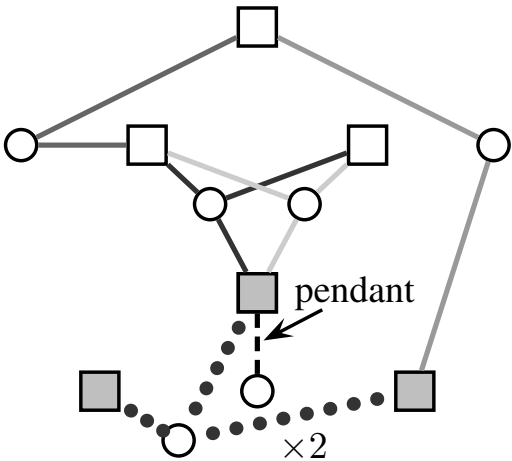

(b) blowup graph $\mathcal{X} \circledast Q-B$ after removal

Figure 2: In $(a): \mathcal{X} \circledast Q$, edges in $B \subseteq E(\mathcal{X})$ in bold, copies of $Q$ are dotted, terminals in $Q$ are filled gray. In $(b)$ : feasible blowup graph $\mathcal{X} \circledast Q-B$ (which is not minimal due to the pendant edge that may also be removed).

in $\Gamma(\mathcal{X})$, this is the same as contracting a component in $\mathcal{K}$ with probability proportional to $x_{\tilde{Q}}$. Again, we allow ourselves to choose an arbitrary distribution over $\mathcal{B}_{Q}$ for removals on contracting $Q$, and ask what probability vectors $p$ describing edge removal probabilities are attainable. But any such probability vector is given by some convex combination $\frac{1}{|\Gamma(\mathcal{X})|} \sum_{Q \in \Gamma(\mathcal{X})} q^{Q}$, where $q^{Q} \in B\left(M_{Q}\right)$. In other words, the attainable probability vectors form precisely the polytope $B_{\text {rem }}$ given by the Minkowski sum

$$
B_{\text {rem }}=\frac{1}{|\Gamma(\mathcal{X})|} \sum_{Q \in \Gamma(\mathcal{X})} B\left(M_{Q}\right) .
$$

This implies that $B_{\text {rem }}$ is a polymatroid [14]; from our knowledge of the rank functions of the $M_{Q}$ 's, we can also describe the rank function of $B_{\text {rem }}$, as will be described in detail in Section 3 .

In the following, we use scaled cost to refer to costs reduced by a factor of $N$, compensating for the blowup factor. The goal is to show that the expected scaled cost of removed edges is large, compared to the expected cost of the component that is contracted. Perfection would be if we could always remove edges of total scaled cost as large as the cost of the contracted component, but of course this is not possible (as it would imply an integrality gap of 1). Thus we lower our goals slightly. It is possible to show that there is a point $p \in B_{\text {rem }}$ with $p_{e} \geq \frac{N}{2|\Gamma(\mathcal{X})|}$ for all $e \in E(\mathcal{X})$. This gives an expected decrease of $\operatorname{cost}(\mathcal{X}) /(2|\Gamma(\mathcal{X})|)$ in the LP solution after scaling down, and the expected cost of the contracted component is $\operatorname{cost}(\mathcal{X}) /|\Gamma(\mathcal{X})|$; so this implies only an uninteresting bound of 2 on the integrality gap. Instead, we must choose the distribution more carefully.

More precisely, we will choose a well-structured subset $K \subseteq$ $E(\mathcal{X})$ and only consider removal probabilities $p \in B_{\text {rem }}$ whose support is contained in $K$. The set $K$ will be chosen to be a minimal subset of $E(\mathcal{X})$ whose removal from $E(\mathcal{X})$ disconnects all terminals in the blowup graph. We call such a set a splitting set ${ }^{1}$. Interestingly, the family of all splitting sets form the bases of a cographic matroid, since $K$ is a splitting set precisely when $E(\mathcal{X}) \backslash K$ is a spanning tree in the graph obtained from $\mathcal{X}$ by contracting together all its terminals. As we will see more formally in the proof of Theorem 2.2, when choosing $K$ to be a splitting set, the set $\mathcal{B}_{Q}^{K}=\left\{B \in \mathcal{B}_{Q} \mid B \subseteq K\right\}$ is nonempty for every $Q$, and so form the bases of the matroid $M_{Q}^{K}$ obtained by restricting $M_{Q}$ to $K$. This implies that the polytope $B_{\text {rem }}^{K}=\left\{p \in B_{\text {rem }} \mid \operatorname{supp}(p) \subseteq K\right\}$ of removal probabilities we consider is nonempty, and thus forms the base polytope of the polymatroid obtained by restricting the polymatroid corresponding to $B_{\text {rem }}$ to $K$.

Once we have chosen some splitting set $K$, we will call edges in $K$ core edges, and all other edges cleanup edges. To see the reason for this name, recall that the matroid $M_{Q}$ describes only

\footnotetext{
${ }^{1}$ The complements of splitting sets are sometimes called losses.
} 


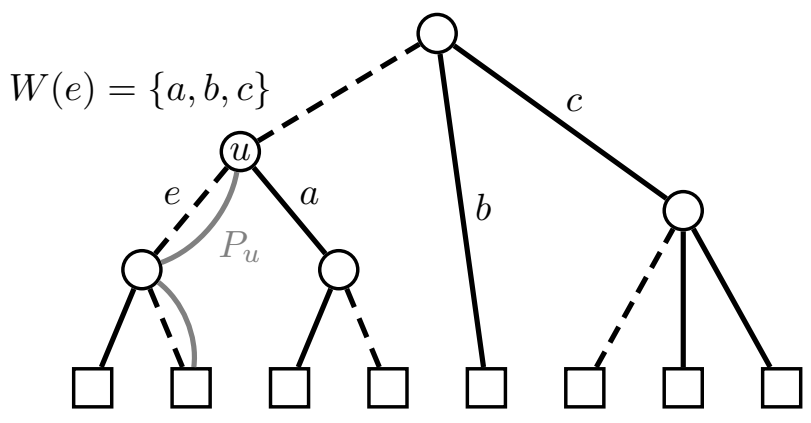

Figure 3: Illustration of the definition of $W(e)$. Depicted is some component $C$ with core edges $K$ (solid) and cleanup edges (dashed).

the minimal edge removals upon contracting $Q$. However, there may be other removals that are possible; for $B \in \mathcal{B}_{Q}$, there may be pendant edges in $(\mathcal{X} \circledast Q)-B$ which can be removed without having any effect on feasibility. Our choice of $K$ ensures that for any edge $e \in E(\mathcal{X}) \backslash K$, e can be deleted ("cleaned up") once enough edges of $K \cap E(C)$ have been removed, where $C$ is the component containing $e$. But just as importantly, we can prove

THEOREM 2.2. If $K$ is any splitting set, then there is a distribution over $\mathcal{B}_{Q}^{K}$ for each $Q \in \Gamma(\mathcal{X})$ such that if $Q$ is chosen uniformly at random from $\Gamma(\mathcal{X})$, and then $B$ is chosen from $\mathcal{B}_{Q}^{K}$ according to the chosen distribution, then

$$
\mathbb{P}\{e \in B\} \geq N /|\Gamma(\mathcal{X})| \quad \text { for each } e \in K .
$$

This is discussed further in Section 3.

\subsection{The algorithm}

For the accounting in our analysis, we will need to keep track of precisely which edges in $E(C) \cap K$ must be removed before an edge $e \in E(C) \backslash K$ can be deleted (cleaned up). Define $W(e) \subseteq K$, the witness set of edge $e$, as the unique minimal set of edges in $K$ such that after removing $W(e), e$ becomes a pendant edge and can be cleaned up. The fact that there exists such a unique set is shown in Lemma B.1 in the appendix. We also define $W(e)=\{e\}$ if $e \in K$. Figure 3 shows an example of a witness set.

We define a weight (distinct from the cost) on all core edges in such a way that the total weight of core edges equals the total cost of $\mathcal{X}$, by charging the cost of a cleanup edge to the core edges in its witness set. More precisely, let

$$
w(e)=c(e)+\sum_{f \notin K: e \in W(f)} \frac{c(f)}{|W(f)|} \quad \text { for all } e \in K .
$$

The following is an easy consequence of Theorem 2.2 and the fact that $\sum_{e \in K} w(e)=\operatorname{cost}(\mathcal{X})$ :

LEMMA 2.3. Let $K$ be any splitting set. There exists some component $Q$ such that $\operatorname{cost}(Q) \leq w\left(B^{Q}\right) / N$, where $B^{Q}$ is a maximum weight basis of $M_{Q}^{K}$.

In the next section in (4) we give an explicit formula for the rank function of $M_{Q}^{K}$ (for arbitrary $Q$ and $K$ ) as the minimum of a submodular function (hence, this provides an independence oracle for $M_{Q}^{K}$ ). This explicit formula is crucial for the analysis of the integrality gap. From a computational point of view, however, we can provide faster ways to compute the rank and find a maximum weight basis of $M_{Q}^{K}$ by giving an explicit description of $M_{Q}$ (and thus also
$M_{Q}^{K}$ ) as a gammoid. This is shown in Appendix A. This gammoid structure obviates the need for submodular function minimization and the greedy procedure to obtain a maximum weight basis. More precisely, determining the rank of $M_{Q}^{K}$ reduces to a single maximum flow computation on an appropriate digraph, and finding a maximum weight basis over $M_{Q}^{K}$ reduces to a minimum-cost flow problem.

We are now ready to describe precisely our deterministic algorithm, given in Algorithm 1. In the algorithm, at each stage we choose a component $Q$ and contract it (in the usual sense, yielding an instance with a smaller vertex set). Thus at intermediate stages of the algorithm, $\mathcal{X}$ will be a feasible blowup graph of some contraction of the original graph $G$. We also emphasize that the witness sets $W(e)$, and hence also the weights $w(e)$, depend on the blowup graph in the particular iteration.

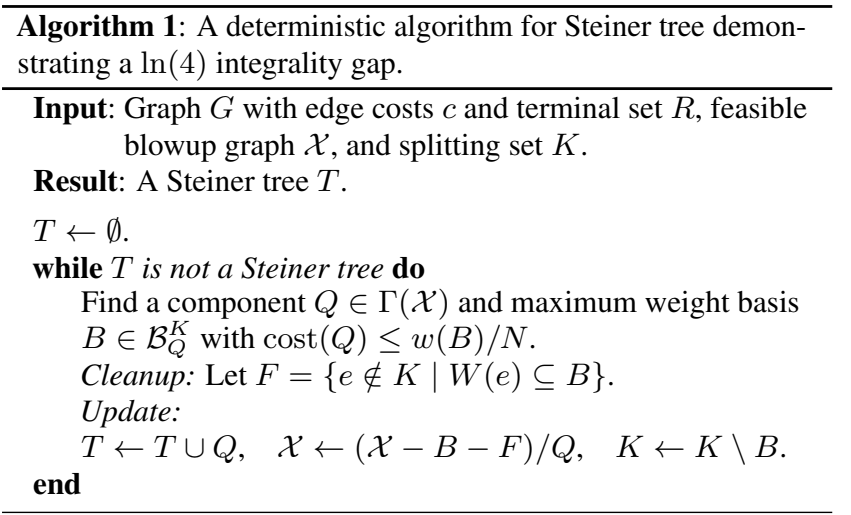

We now define, for any blowup graph $\mathcal{X}$ and splitting set $K$, a potential function $\Phi_{K}(\mathcal{X})$ by

$$
\Phi_{K}(\mathcal{X}):=\sum_{e \in E(\mathcal{X})} c(e) H(|W(e)|)
$$

where $H(\ell):=1+1 / 2+\cdots+1 / \ell$ is the harmonic function.

THEOREM 2.4. For any minimal splitting set $K$ and feasible blowup graph $\mathcal{X}$, Algorithm 1 yields a solution of cost at most $\Phi_{K}(\mathcal{X}) / N$.

The proof of this theorem (given in Appendix B) essentially boils down to showing that in a single step of the algorithm, the expected cost of the contracted component is no larger than the decrease in the potential function scaled down by $1 / N$. Let $\mathcal{X}_{t}$ and $K_{t}$ be the blowup graph and splitting set at iteration $t$ of the algorithm, with $B_{t}$ the selected removal set. We are able to show that $\Phi_{K_{t}}\left(\mathcal{X}_{t}\right)-$ $\Phi_{K_{t+1}}\left(\mathcal{X}_{t+1}\right) \geq w\left(B_{t}\right)$, from which the theorem immediately follows.

From this, we can use an averaging argument to show the $\ln (4)$ integrality gap bound. Essentially, if $K$ is chosen randomly from the matroid of possible minimal splitting sets according to an appropriate distribution, it can be shown that

$$
\mathbb{E}\left\{\Phi_{K}(\mathcal{X})\right\} \leq \ln (4) \cdot \operatorname{cost}(\mathcal{X}) .
$$

It is also possible to minimize $\Phi_{K}(\mathcal{X})$ as a function of $K$, via a dynamic program. The full proof can be found in the appendix: altogether we obtain, recalling $\operatorname{cost}(\mathcal{X})=N \cdot \operatorname{cost}(x)$,

THEOREM 2.5. For any solution $x$ of (LP), and choosing $K$ to minimize $\Phi_{K}(\mathcal{X})$, Algorithm 1 returns a solution of cost at most $\ln (4) \cdot \operatorname{cost}(x)$. 
We emphasize again that while we have described everything in terms of the blowup graph, it is possible to implement Algorithm 1 directly in terms of the LP solution, yielding a polynomial time algorithm. Details will be provided in the full version.

\section{DEEPER INTO THE MATROID STRUC- TURE}

In this section, we discuss in more detail the heart of our arguments: uncovering the matroid structure of edge removals, and showing appropriate uniform removal probabilities after the random contraction of a component.

In what follows, we will often need to refer to the terminal set of a component $C$, so we will again abuse notation and write, e.g., $|C|$ for the number of terminals in $C$. Define $h_{\mathcal{X}}: 2^{R} \backslash\{\emptyset\} \rightarrow \mathbb{N}$ by

$$
h_{\mathcal{X}}(S)=N(|S|-1)-\sum_{C \in \Gamma(\mathcal{X})}(|S \cap C|-1)^{+} .
$$

It is immediate from (LP) that $\mathcal{X}$ is feasible if and only if

$$
h_{\mathcal{X}}(S) \geq 0 \quad \forall S \subseteq R, S \neq \emptyset \quad \text { and } \quad h_{\mathcal{X}}(R)=0 .
$$

Indeed, $h_{\mathcal{X}}(S)$ is, up to scaling, simply the slack (or if negative, violation) of the corresponding constraint in (LP). Two important properties of $h_{\mathcal{X}}$ are the following:

\section{LEMMA 3.1. For any blowup graph $\mathcal{X}$,}

i) $h_{\mathcal{X}}$ is intersecting submodular, i.e.,

$$
h_{\mathcal{X}}\left(S_{1} \cup S_{2}\right)+h_{\mathcal{X}}\left(S_{1} \cap S_{2}\right) \leq h_{\mathcal{X}}\left(S_{1}\right)+h_{\mathcal{X}}\left(S_{2}\right)
$$

for any two sets $S_{1}, S_{2} \subseteq R$ with $S_{1} \cap S_{2} \neq \emptyset$; and

ii) for any $F \subseteq E(\mathcal{X})$ and $\emptyset \neq S \subseteq R$,

$$
h_{\mathcal{X}}(S) \leq h_{\mathcal{X}-F}(S) \leq h_{\mathcal{X}}(S)+|F| .
$$

Proof. i) This follows immediately from the fact that for any $C \subseteq R$, the function $S \rightarrow(|S \cap C|-1)^{+}$is intersecting supermodular.

ii) The removal of any additional edge $e \in E(\mathcal{X})$ from $\mathcal{X}$ leads to a split of some component $C$ of $\mathcal{X}$ into subcomponents $C_{1}, C_{2}$ with $C_{1} \cap C_{2}=\emptyset, C_{1} \cup C_{2}=C$. Hence,

$$
\begin{aligned}
& h_{\mathcal{X}-e}(S)-h_{\mathcal{X}}(S) \\
& \quad=(|S \cap C|-1)^{+}-\left(\left|S \cap C_{1}\right|-1\right)^{+}-\left(\left|S \cap C_{2}\right|-1\right)^{+}
\end{aligned}
$$

lies in $\{0,1\}$, which leads to $h_{\mathcal{X}}(S) \leq h_{\mathcal{X}-e}(S) \leq h_{\mathcal{X}}(S)+1$. Applying this repeatedly yields the claim.

An interesting consequence, that essentially follows by intersecting submodularity of $h_{\mathcal{X}}$ and standard uncrossing techniques, is that any basic feasible solution $\left(x_{C}\right)_{C \in \mathcal{K}}$ to (LP) has a support of size bounded by $|R|-1$. More precisely, one can show that any basic feasible solution to (LP) can be defined by tight non-negativity constraints $\left(x_{C} \geq 0\right)$ together with a family of tight constraints of type $\sum_{C \in \mathcal{K}} x_{C}(|S \cap C|-1)^{+} \leq|S|-1$ for a laminar family of sets $S \subseteq R$ with $|S| \geq 2$. A bound of $|R|-1$ on the support of $\left(x_{C}\right)_{C \in \mathcal{K}}$ then readily follows (see [10] for an example of this reasoning). For an equivalent version of (LP), this result was already obtained through a rather involved technique by Chakrabarty et al. [6].

For convenience, define

$$
h_{\bar{F}}(S):=h_{\mathcal{X}-F}(S)=N(|S|-1)-\sum_{C \in \Gamma(\mathcal{X}-F)}(|S \cap C|-1)^{+} .
$$

The following lemma describes feasibility of $(\mathcal{X} \circledast Q)-F$ in a convenient form, and also shows that we need only consider constraints corresponding to subsets containing $Q$.

LEMMA 3.2. The blowup graph $(\mathcal{X} \circledast Q)-F$ is feasible if and only if $h_{\bar{F}}(R)=N(|Q|-1)$ and $h_{\bar{F}}(S) \geq N(|Q|-1)$ for all $S \supseteq Q$.

Proof. Let $\mathcal{X}^{\prime}=(\mathcal{X} \circledast Q)-F$. Then $\mathcal{X}^{\prime}$ is feasible iff $h_{\mathcal{X}^{\prime}}(S) \geq 0$ for all $S \subseteq R, S \neq \emptyset$, with equality for $S=R$. But

$$
h_{\mathcal{X}^{\prime}}(S)=h_{\bar{F}}(S)-N(|S \cap Q|-1)^{+},
$$

and so this can be equivalently stated as $h_{\bar{F}}(S) \geq N(|S \cap Q|-1)^{+}$ for all $S \neq \emptyset$, and $h_{\bar{F}}(R)=N(|Q|-1)$.

All that needs to be proved then is that only the constraints for $S \supseteq Q$ need to be considered. So suppose $S$ is a violated set: $h_{\mathcal{X}^{\prime}}(S)<0$. Then $S \cap Q \neq \emptyset$, otherwise $h_{\mathcal{X}^{\prime}}(S)=h_{\bar{F}}(S) \geq$ $h_{\mathcal{X}}(S) \geq 0$ by feasibility of $\mathcal{X}$. But for any such $S$,

$N(|S|-1)-N(|S \cap Q|-1)^{+}=N(|S \cup Q|-1)-N(|Q|-1)^{+}$

and clearly

$\sum_{C \in \Gamma(\mathcal{X}-F)}(|S \cap C|-1)^{+} \leq \sum_{C \in \Gamma(\mathcal{X}-F)}(|(S \cup Q) \cap C|-1)^{+}$.

Subtracting the last two lines, using (3) and the definition of $h_{\bar{F}}(\cdot)$, we obtain that $h_{\mathcal{X}^{\prime}}(S \cup Q) \leq h_{\mathcal{X}^{\prime}}(S)$. Since $S$ was a violating set, so is $S \cup Q$.

$$
\begin{aligned}
\text { Define } r_{Q}: 2^{E(\mathcal{X})} & \rightarrow \mathbb{N} \text { by } \\
& r_{Q}(F)=\min _{S \supseteq Q} h_{\bar{F}}(S) .
\end{aligned}
$$

We will show:

PROPOSITION 3.3. The function $r_{Q}$ is the rank function of $a$ matroid of rank $N(|Q|-1)$.

Once we have this, it is straightforward to show that this matroid precisely describes the minimal edge removals:

THEOREM 3.4. The set of bases of the matroid defined by $r_{Q}$ is precisely $\mathcal{B}_{Q}$.

Proof. Let $\mathcal{B}_{Q}^{\prime}$ be the set of bases of the matroid defined by $r_{Q}$, and consider any $B \in \mathcal{B}_{Q}^{\prime}$. By the definition of $r_{Q}$, we have that

$$
h_{\bar{B}}(S) \geq r_{Q}(B)=N(|Q|-1) \quad \text { for any } S \supseteq Q .
$$

Moreover, by Lemma 3.1 (ii),

$$
h_{\bar{B}}(R) \leq h_{\mathcal{X}}(R)+|B|=N(|Q|-1) ;
$$

the final equality follows since $|B|=r_{Q}(B)=N(|Q|-1)$ and $h_{\mathcal{X}}(R)=0$ by feasibility of $\mathcal{X}$. Thus by Lemma $3.2,(\mathcal{X} \circledast Q)-B$ is feasible.

Conversely, consider any $B \in \mathcal{B}_{Q}$. By feasibility and Lemma 3.2 again, $h_{\bar{B}}(S) \geq N(|Q|-1)$ for all $S \supseteq Q$, with equality for $S=R$. Thus $r_{Q}(B)=N(|Q|-1)$, and so there is some $B^{\prime} \subseteq B$ with $B^{\prime} \in \mathcal{B}_{Q}^{\prime}$. But then $B^{\prime}$ is also a feasible removal set by the above, and so by minimality $B^{\prime}=B$.

Proof of Proposition 3.3. First, observe from (1) applied to the empty blowup graph that

$$
r_{Q}(E(\mathcal{X}))=\min _{S \supseteq Q} N(|S|-1)=N(|Q|-1) .
$$

We must show that $r_{Q}$ is increasing, submodular, and satisfies $r_{Q}(F) \leq|F|$ for all $F \subseteq E(\mathcal{X})$. The fact that $r_{Q}$ is increasing 
follows immediately from the definitions of $r_{Q}$ and $h_{\bar{F}}$; removing a larger set can only increase the slack. Considering some fixed $S \supseteq Q$, we have by Lemma 3.1 (ii) that $h_{\mathcal{X}-F}(S) \leq h_{\mathcal{X}}(S)+|F|$. Thus $r_{Q}(F) \leq r_{Q}(\emptyset)+|F|=|F|$ since $r_{Q}(\emptyset)=0$ by feasibility of $\mathcal{X}$.

Now we come to the main part of the proof, showing that $r_{Q}$ is submodular. We must show that for any $F_{1} \subseteq F_{2} \subseteq E(\mathcal{X})$ and $e \notin F_{2}$,

$$
r_{Q}\left(F_{1}+e\right)-r_{Q}\left(F_{1}\right) \geq r_{Q}\left(F_{2}+e\right)-r_{Q}\left(F_{2}\right) .
$$

It is clearly sufficient to show this for $F_{1}$ and $F_{2}$ differing by a single edge. Consider any $S \supseteq Q$ and $i \in\{1,2\}$. The difference

$$
\begin{aligned}
& h_{\overline{F_{i}+e}}(S)-h_{\bar{F}_{i}}(S) \\
& \quad=\sum_{C \in \Gamma\left(\mathcal{X}-F_{i}\right)}(|C \cap S|-1)^{+}-\sum_{C \in \Gamma\left(\mathcal{X}-\left(F_{i}+e\right)\right)}(|C \cap S|-1)^{+}
\end{aligned}
$$

is one or zero, and it is one precisely if $e$ splits up some component $C \in \Gamma\left(\mathcal{X}-F_{i}\right)$ into two components $C_{1}, C_{2}$ that both intersect $S$. If this is the case for some component in $\Gamma\left(\mathcal{X}-F_{2}\right)$, then $e$ will also split up some component in $\Gamma\left(\mathcal{X}-F_{1}\right)$ into two pieces both intersecting $S$, since $\mathcal{X}-F_{2}$ is a subgraph of $\mathcal{X}-F_{1}$. Thus for any $S \supseteq Q$,

$$
h_{\overline{F_{2}+e}}(S)-h_{\bar{F}_{2}}(S) \leq h_{\overline{F_{1}+e}}(S)-h_{\bar{F}_{1}}(S) .
$$

It also follows that for any $S, S^{\prime} \subseteq R$ with $Q \subseteq S \subseteq S^{\prime}$,

$$
h_{\overline{F_{1}+e}}(S)-h_{\bar{F}_{1}}(S) \leq h_{\overline{F_{1}}+e}\left(S^{\prime}\right)-h_{\bar{F}_{1}}\left(S^{\prime}\right) .
$$

Let $\mathcal{S}_{i}$ be the set of terminal subsets containing $Q$ that minimize $h_{\bar{F}_{i}}(S)$, over all $S \supseteq Q$. Since $h_{\bar{F}_{1}}$ is intersecting submodular by Lemma 3.1, there is a unique maximal set $S_{1}^{*} \in \mathcal{S}_{1}$, meaning $S_{1}^{*} \supseteq S$ for all $S \in \mathcal{S}_{1}$. Similarly, there is a unique minimal set $S_{2}^{*} \in \mathcal{S}_{2}$; so $S_{2}^{*} \subseteq S$ for all $S \in \mathcal{S}_{2}$. We first show $S_{2}^{*} \subseteq S_{1}^{*}$. Notice that for $S \supseteq Q$ with $S \notin \mathcal{S}_{1}$ we have $h_{\bar{F}_{2}}(S) \geq h_{\bar{F}_{1}}(S) \geq$ $h_{\bar{F}_{1}}\left(S_{1}^{*}\right)+1$, where the first inequality follows by Lemma 3.1 (ii). Furthermore, $h_{\bar{F}_{2}}\left(S_{1}^{*}\right) \leq h_{\bar{F}_{1}}\left(S_{1}^{*}\right)+1$, again by Lemma 3.1 (ii). Hence $h_{\bar{F}_{2}}\left(S_{1}^{*}\right) \leq h_{\bar{F}_{2}}(S) \forall S \supseteq Q, S \notin \mathcal{S}_{1}$, and thus $\mathcal{S}_{1}$ must contain some minimizers of $h_{\overline{F_{2}}}$, i.e., $\mathcal{S}_{1} \cap \mathcal{S}_{2} \neq \emptyset$. Since $S_{2}^{*}$ is the minimal set in $\mathcal{S}_{2}$ and $S_{1}^{*}$ is the maximal set in $\mathcal{S}_{1}$ we obtain $S_{2}^{*} \subseteq S_{1}^{*}$.

We finally have

$$
\begin{aligned}
& r_{Q}\left(F_{2}+e\right)-r_{Q}\left(F_{2}\right) \\
& \quad=h_{\overline{F_{2}+e}}\left(S_{2}^{*}\right)-h_{\overline{F_{2}}}\left(S_{2}^{*}\right) \\
& \quad \leq h_{\overline{F_{1}+e}}\left(S_{2}^{*}\right)-h_{\overline{F_{1}}}\left(S_{2}^{*}\right) \quad \text { by }(6) \\
& \quad \leq h_{\overline{F_{1}+e}}\left(S_{1}^{*}\right)-h_{\overline{F_{1}}}\left(S_{1}^{*}\right) \quad \text { by }(7), \text { since } S_{2}^{*} \subseteq S_{1}^{*} \\
& \quad=r_{Q}\left(F_{1}+e\right)-r_{Q}\left(F_{1}\right) . \quad \square
\end{aligned}
$$

\section{Proof of Theorem 2.2}

We first show that for any component $Q$,

$$
r_{Q}(K)=N(|Q|-1) .
$$

This in turn implies that $\mathcal{B}_{Q}=\left\{B \in \mathcal{B}_{Q} \mid B \subseteq K\right\}$ is nonempty, since the rank of $M_{Q}$ is $N(|Q|-1)$ by Proposition 3.3, and so $B_{\text {rem }} \neq \emptyset$. Notice that for $S \subseteq R, S \neq \emptyset$, we have $h_{K}(S)=$ $N(|S|-1)$, since in $\mathcal{X}-K$ all components contain precisely one terminal. Hence,

$$
r_{Q}(K)=\min _{S \supseteq Q} h_{K}(S)=N(|Q|-1) .
$$

As already discussed in Section 2, the polytope $B_{\text {rem }}$ is simply a weighted Minkowski sum of the base polytopes $B\left(M_{Q}\right)$ for $Q \in \Gamma(\mathcal{X})$. It is well known that the Minkowski sum of matroid polytopes is a polymatroid, and moreover, the rank function of the sum is simply the sum of the rank functions of the summands [14]. Thus, $B_{\text {rem }}$ is the base polytope of a polymatroid with rank function

$$
r=\frac{1}{|\Gamma(\mathcal{X})|} \sum_{Q \in \Gamma(\mathcal{X})} r_{Q} .
$$

To show that the point $p$ given by $p_{e}=N /|\Gamma(\mathcal{X})|$ for all $e \in K$ is in $B_{\text {rem }}$, we need to show that $r(F) \geq|F| \cdot N /|\Gamma(\mathcal{X})|$ for every $F \subseteq K$. Expanding out (8) and the definition of $r_{Q}$, and writing $S_{Q}$ for the subset $S \supseteq Q$ that attains the minimum in (4), we obtain

$$
r(F)=\frac{1}{|\Gamma(\mathcal{X})|} \sum_{Q \in \Gamma(\mathcal{X})} h_{\bar{F}}\left(S_{Q}\right) .
$$

We now observe that because $K$ is a splitting set, $h_{\bar{F}}(R)=|F|$. For imagine removing the edges of $F$ from $\mathcal{X}$ one by one; $h_{\mathcal{X}-F}(R)-$ $h_{\mathcal{X}}(R)$ just counts the number of times where a component is split by the deleted edge in this process. But by the nature of minimal splitting sets, this must happen at every step-no pendant edges are formed at any stage. Hence $h_{\bar{F}}(R)-h_{\mathcal{X}}(R)=|F|$; moreover, $h_{\mathcal{X}}(R)=0$ by feasibility, so indeed $h_{\bar{F}}(R)=|F|$. Thus to finish the proof, it suffices to show

Claim 3.5. $\sum_{Q \in \Gamma(\mathcal{X})} h_{\bar{F}}\left(S_{Q}\right) \geq N \cdot h_{\bar{F}}(R)$.

To prove Claim 3.5, we replace the function $h_{\bar{F}}$ on the left-hand side of the inequality by a function $f$ that lower bounds $h_{\bar{F}}$ and is well structured. More precisely, $f$ is chosen to be a conic combination of a special type of intersecting submodular functions which we call partition functions: for any partition $\mathcal{P}=\left\{P_{1}, \ldots, P_{n}\right\}$ of $R$, the corresponding partition function $f_{\mathcal{P}}$ is given by

$$
f_{\mathcal{P}}(S)=\left(\left|\left\{j \in[n] \mid P_{j} \cap S \neq \emptyset\right\}\right|-1\right)^{+} \quad \forall S \subseteq R .
$$

The following theorem (whose proof can be found in Appendix C) guarantees the existence of the function $f$ that we need to prove Claim 3.5.

THEOREM 3.6. Let $h: 2^{U} \rightarrow \mathbb{R}_{+}$any nonnegative intersecting submodular function with $h(\{v\})=0$ for all $v \in U$. Then there is a monotone intersecting submodular function $f$ of the form

$$
f=\sum_{i=1}^{k} \lambda_{i} f_{\mathcal{P}^{i}}
$$

for some $k \in \mathbb{N}$, where $\lambda_{i}>0$ and $\mathcal{P}^{i}$ is a partition of $U$ for each $1 \leq i \leq k$, satisfying:

i) $f(S) \leq h(S)$ for all $S \subseteq U$, and

ii) $f(U)=h(U)$.

Consider the function $h_{\bar{F}}^{+}$defined by $h_{\bar{F}}^{+}(S)=\max \left\{h_{\bar{F}}(S), 0\right\}$. Then $h_{\bar{F}}^{+}$differs from $h_{\bar{F}}$ only on the empty set, since $h_{\bar{F}}(S) \geq$ 0 for all $S \neq \emptyset$. Thus $h_{\bar{F}}^{+}$is still intersecting submodular, and also nonnegative. Furthermore, $h_{\bar{F}}^{+}(\{v\})=0$ for any $v \in R$ by definition of $h_{\bar{F}}$. Let $f=\sum_{i=1}^{k} \lambda_{i} f_{\mathcal{P}^{i}}$ be the function obtained by 
applying Theorem 3.6 to $h_{\bar{F}}^{+}$. We then have

$$
\begin{aligned}
\sum_{Q \in \Gamma(\mathcal{X})} h_{\bar{F}}\left(S_{Q}\right) & \geq \sum_{Q \in \Gamma(\mathcal{X})} f\left(S_{Q}\right) \\
& \geq \sum_{Q \in \Gamma(\mathcal{X})} f(Q) \\
& =\sum_{Q \in \Gamma(\mathcal{X})} \sum_{i=1}^{k} \lambda_{i} f_{\mathcal{P}_{i}}(Q) \\
& =\sum_{i=1}^{k} \lambda_{i} \sum_{Q \in \Gamma(\mathcal{X})} f_{\mathcal{P}_{i}}(Q),
\end{aligned}
$$

where the first inequality holds since $h_{\bar{F}}(S)=h_{\bar{F}}^{+}(S) \geq f(S)$ for all $S \neq \emptyset$, and the second inequality holds since $f$ is monotone and $Q \subseteq S_{Q}$.

As observed by Chakrabarty et al. [6], any solution $x$ to (LP) satisfies the following partition constraints for any partition $\mathcal{P}$ of $R$ :

$$
\sum_{C \in \mathcal{K}} x_{C} f_{\mathcal{P}}(C) \geq|\mathcal{P}|-1
$$

where $|\mathcal{P}|$ is the number of sets in partition $\mathcal{P}$. In our blown-up setting this translates into

$$
\sum_{Q \in \Gamma(\mathcal{X})} f_{\mathcal{P}}(Q) \geq N(|\mathcal{P}|-1) .
$$

Combining this observation with (9) and using $\left|\mathcal{P}_{i}\right|-1=f_{\mathcal{P}_{i}}(R)$, Claim 3.5 follows since

$$
\begin{aligned}
\sum_{Q \in \Gamma(\mathcal{X})} h_{\bar{F}}\left(S_{Q}\right) & \geq \sum_{i=1}^{k} \lambda_{i} \sum_{Q \in \Gamma(\mathcal{X})} f_{\mathcal{P}_{i}}(Q) \geq \sum_{i=1}^{k} \lambda_{i} N\left(\left|\mathcal{P}_{i}\right|-1\right) \\
& =N \cdot \sum_{i=1}^{k} \lambda_{i} f_{\mathcal{P}_{i}}(R)=N \cdot f(R)=N \cdot h_{\bar{F}}(R),
\end{aligned}
$$

where the last equality follows from property (ii) of Theorem 3.6.

\section{QUASI-BIPARTITE GRAPHS}

A Steiner tree instance is called quasi-bipartite if there are no edges between Steiner vertices; in other words, all components are either stars or single edges. The situation is much simplified in this case, and we can obtain a better approximation factor. We prove the following:

LEMMA 4.1. Consider a quasi-bipartite instance. Let $K=$ $E(\mathcal{X}) \backslash E_{\min }$, where $E_{\min }$ consists of a cheapest edge from every component. Then

$$
\Phi_{K}(\mathcal{X}) \leq \frac{73}{60} \cdot \operatorname{cost}(\mathcal{X})
$$

Together with Theorem 2.4, Lemma 4.1 implies a $73 / 60<1.217$ bound on the integrality gap of (LP) for quasi-bipartite graphs. This again matches the currently best approximation algorithm for this problem [3].

Proof of Lemma 4.1. For any component $C$, we have that all edges $e$ of $C$ have $|W(e)|=1$ except for the cheapest edge $e_{\min }$ for which $\left|W\left(e_{\min }\right)\right|=|C|-1$. Therefore,

$$
\begin{aligned}
& \sum_{e \in E(C)} c(e) H(|W(e)|) \\
& =c\left(e_{\min }\right) H(|C|-1)+\sum_{e \in E(C), e \neq e_{\min }} c(e) \\
& \leq \sum_{e \in E(C)} c(e) \frac{|C|-1+H(|C|-1)}{|C|} \\
& \leq \frac{73}{60} \sum_{e \in E(C)} c(e)
\end{aligned}
$$

since $\max _{k \geq 2, k \in \mathbb{N}} \frac{k-1+H(k-1)}{k}=\frac{73}{60}$ (which is attained for $k=$ 5). Summing over all components gives the desired inequality.

One of the major drawbacks of relying on (LP), or any of the hypergraphic LPs, is that solving them is computational intensive; in general, to obtain a $1+\epsilon$ approximation, nothing better than $n^{2^{\Omega(1 / \epsilon)}}$ time is known. This can be improved somewhat to $n^{\Omega(1 / \epsilon)}$ in quasibipartite graphs, but this is still very slow. We show how to sidestep this issue and obtain a reasonable running time for quasi-bipartite graphs by instead solving the much more tractable bidirected cut relaxation. Combined with the fact that we do not need to re-solve the LP in each iteration, we obtain a markedly faster algorithm than the one of Byrka et al. [3].

More precisely, we show how a solution to the bidirected cut relaxation can be transformed into a solution to (LP) with the same cost, via a natural greedy procedure for quasi-bipartite instances. Previously, [6] showed that the bidirected cut relaxation always has the same objective value as the hypergraphic relaxations, and this was done by lifting an optimum dual solution for the partition-based relaxation (which is equivalent to (LP) even in general graphs [6]) to a dual solution of $(\mathrm{BCR}(r))$. However, the authors of [6] posed as an open question: for a given optimum bidirected cut solution, can a corresponding primal solution to (LP) be directly extracted without solving (LP)? We answer this question affirmatively.

Let $G=(V, E)$ be a quasi-bipartite graph, and let $\vec{E}$ be the bidirection of $E$, i.e., for any $\{u, v\} \in E, \vec{E}$ contains $\operatorname{arcs}(u, v)$ and $(v, u)$. The bidirected cut relaxation with root terminal $r \in R$ is

$$
\begin{aligned}
\min \sum_{e \in \vec{E}} c_{e} x_{e} & \\
x\left(\delta^{+}(S)\right) \geq 1 \quad & \forall S \subseteq V \backslash\{r\}, S \cap R \neq \emptyset \\
x_{e} \geq 0 & \forall e \in \vec{E}
\end{aligned}
$$

$(\operatorname{BCR}(r))$

In words: we need to reserve enough capacity in order to support a unit flow from every terminal to the current root $r$.

To avoid an unnecessary case analysis, we split edges between terminals by inserting a dummy Steiner vertex (we split the edge cost arbitrarily among the two parts). Let $x$ be an optimum solution to $(\operatorname{BCR}(r))$; then the natural decomposition is as follows. For a star with center $u \in V \backslash R$, take an $\operatorname{arc}(u, s)$ with positive outgoing flow and all $\operatorname{arcs} H=\{(t, u) \mid x(t, u)>0 ; t \neq s\}$ carrying incoming flow. Let $\epsilon$ be the minimum capacity on any of these arcs. Then transfer this capacity into a component $\{s\} \cup\{t \mid(t, u) \in H\}$. Iterate this process until all capacity has been transferred. The main result of this section is:

THEOREM 4.2. Let $x$ be an optimum solution for $(\mathrm{BCR}(r))$. Then the natural decomposition yields a feasible optimum solution for (LP) with the same objective value. 
Imagine that we want to "relocate" the root from $r$ to another terminal $r^{\prime}$. We can do this by considering a unit flow from $r^{\prime}$ to $r$, and reversing all capacity corresponding to this flow. This provides a feasible solution for $\operatorname{BCR}\left(r^{\prime}\right)$ that we term $x^{\left(r^{\prime}\right)}$, which again has the same cost (see [11] for a much more complicated proof). Note that for any $\{u, v\} \in E$, the $\operatorname{sum} x^{(r)}(u, v)+x^{(r)}(v, u)$ is independent of $r$, and we denote this sum by $x(\{u, v\})$. For a Steiner vertex $u \in V \backslash R$, let $N(u):=\{v \mid\{u, v\} \in E, x(\{u, v\})>0\}$ be the set of neighbours in the star with center $u$. It suffices to show Theorem 4.2 for basic solutions, since the decomposition of a convex combination of capacity vectors equals the convex combination of natural decompositions. By standard arguments, we may assume that the edge costs are distinct for all edges in the same star.

LEMMA 4.3. In a star with center $u$ and $r \in N(u)$ one has $x^{(r)}(r, u)=0$ and $x^{(r)}(u, s)=0$ for each $s \in N(u)$ with $c(u, s)>c(u, r)$.

PROOF. The flow on arc $(r, u)$ can be removed and the flow on $(u, s)$ arc can be redirected to $(u, r)$. Both operations would leave the solution feasible and decrease the cost, contradicting optimality.

See Figure 4 for an illustration of the claim.

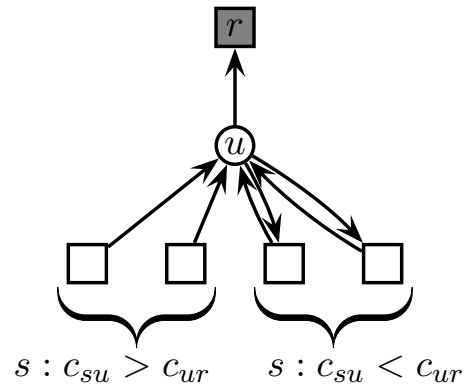

Figure 4: Arcs in the optimum solution $x^{(r)}$ that may carry positive flow.

Observe that if we choose $r=\operatorname{argmin}\{c(u, r) \mid r \in N(u)\}$, then $x^{(r)}(u, s)=0$ for all $s \in N(u) \backslash\{r\}$. Next, we consider one iteration of the natural decomposition for a star with center $u$, for this choice of the root. For this purpose, insert an extra Steiner vertex $\bar{u}$ into the graph, which has an edge $\{\bar{u}, s\}$ for $s \in N(u)$. For $e=(u, s)$, we abbreviate $\bar{e}=(\bar{u}, s)$ (see Figure 5). We define $c(\bar{e}):=c(e)$ and $x^{(r)}(e)=0$ for all $e \in \delta(\bar{u})$. Note that $x^{(r)}$ is still an optimum solution.

LEMMA 4.4. Let $r:=\operatorname{argmin}\{c(u, r) \mid r \in N(u)\}, H:=$ $\{(u, r)\} \cup\{(s, u) \mid s \in N(u) \backslash\{r\}\}$ and $\varepsilon:=\min \left\{x^{(r)}(e) \mid\right.$ $e \in H\}>0$. Starting from $x^{(r)}$, transfer capacity of $\varepsilon$ from all arcs $e \in H$ to $\bar{e}$ and term the new capacity reservation $\bar{x}^{(r)}$. Then the new capacity vector $\bar{x}^{(r)}$ is a feasible optimum solution for (BCR $(r))$.

PROOF. Our observation in the previous paragraph together with our choice of the root imply that the arcs in $H$ are the only ones incident to $u$ carrying positive flow. Therefore $\varepsilon$ as defined is strictly positive.

We first show that the claim holds for some $\varepsilon>0$ which is small enough. As the cost of the solution does not change, we only need to prove feasibility of $\bar{x}^{(r)}$. Consider any cut $S \subseteq V \backslash\{r\}$ and assume for the sake of a contradiction that $\bar{x}^{(r)}\left(\delta^{+}(S)\right)<1$. For $\varepsilon>0$ small enough, this may only happen if $S$ was a tight cut before, i.e. $x^{(r)}\left(\delta^{+}(S)\right)=1$. Furthermore, any such critical cut must contain at least two arcs of the form $(s, u)$, i.e., $|N(u) \cap S| \geq 2$; otherwise, the cut would still be satisfied after our transfer of capacity. Pick $r^{\prime}:=\operatorname{argmin}\left\{c\left(u, r^{\prime}\right) \mid r^{\prime} \in N(u) \cap S\right\}$. Since $x^{(r)}\left(\delta^{+}(S)\right)=1$, the unit flow from $r^{\prime}$ to $r$ needs all capacities on $(s, u)$ arcs for $s \in N(u) \cap S$. Consequently, when relocating the root to $r^{\prime}$, the flow on all these arcs must be turned around completely. In particular $x^{\left(r^{\prime}\right)}(u, s)>0$ for all $s \in N(u) \cap S$, contradicting Lemma 4.3 by our choice of $r^{\prime}$ and $|N(u) \cap S| \geq 2$. We conclude that we can choose some $\varepsilon>0$ s.t. $\bar{x}^{(r)}$ is feasible. But the argument above shows that no cut $S$ can become tight, thus the only limitation on $\varepsilon$ is the arc capacity. The claim then follows.

We apply Lemma 4.4 iteratively to all stars (in this process we keep relocating the root), adding copies of Steiner vertices as required, until we have a solution $x^{*}$ (with root $r^{*} \in R$ chosen arbitrarily) where (i) every Steiner vertex has flow on at most one outgoing arc, and (ii) the flow on all arcs of a star carrying a nonzero amount of flow is the same. Then $x^{*}$ induces a solution to the directed component-based relaxation:

$$
\begin{aligned}
\min \sum_{C \in \mathcal{K}, s \in C} \operatorname{cost}(C) \cdot y_{C, s} & \\
\sum_{C \in \mathcal{K}, s \in C: C \cap S \neq \emptyset, s \notin S} y_{C, s} & \geq 1 \quad \forall \emptyset \subsetneq S \subseteq R \backslash\left\{r^{*}\right\} \\
y_{C, s} & \geq 0 \quad \forall C \in \mathcal{K} \forall s \in C .
\end{aligned}
$$

The solution $y^{*}$ corresponding to $x^{*}$ is obtained by setting, for each flow-carrying star with terminal set $C$ and outgoing flow on arc $(u, s), y_{C, s}^{*}=x^{*}(u, s)$ (the common flow value in the star). All other components of $y^{*}$ are zero. It is easily checked that $y^{*}$ is feasible, and has the same cost as $x^{*}$ (and hence $x$ ). Then projecting to the undirected formulation, the vector $\left(\sum_{s \in C} y_{C, s}\right)_{C \in \mathcal{K}}$ is feasible for (LP) (see [18]), and moreover corresponds precisely to the natural decomposition described earlier.

\section{REFERENCES}

[1] M. Bern and P. Plassmann. The Steiner problem with edge lengths 1 and 2. Information Processing Letters, 32(4):171-176, 1989.

[2] A. Borchers and D.-Z. Du. The $k$-Steiner ratio in graphs. SIAM Journal on Computing, 26(3):857-869, June 1997.

[3] J. Byrka, F. Grandoni, T. Rothvoß, and L. Sanità. Steiner tree approximation via iterative randomized rounding. Journal of the ACM. To appear.

[4] J. Byrka, F. Grandoni, T. Rothvoß, and L. Sanità. An improved LP-based approximation for Steiner Tree. In Proceedings of the 42nd Annual ACM Symposium on Theory of Computing (STOC), pages 583-592, 2010.

[5] D. Chakrabarty, N. R. Devanur, and V. V. Vazirani. New geometry-inspired relaxations and algorithms for the metric Steiner tree problem. In International Conference on Integer Programming and Combinatorial Optimization (IPCO), pages 344-358, 2008.

[6] D. Chakrabarty, J. Könemann, and D. Pritchard. Hypergraphic LP relaxations for Steiner trees. In International Conference on Integer Programming and Combinatorial Optimization (IPCO). 2010.

[7] D. Chakrabarty, J. Könemann, and D. Pritchard. Integrality gap of the hypergraphic relaxation of Steiner trees: A short 


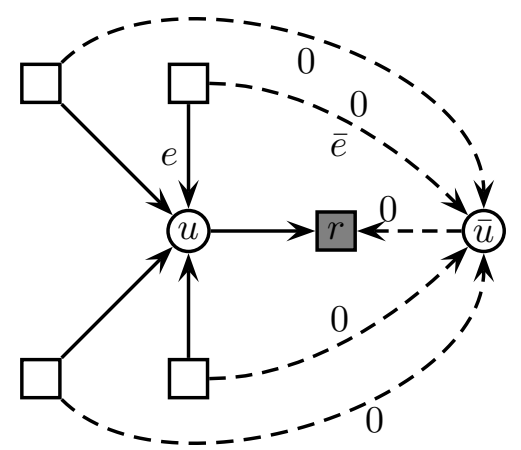

(a) capacities $x^{(r)}$

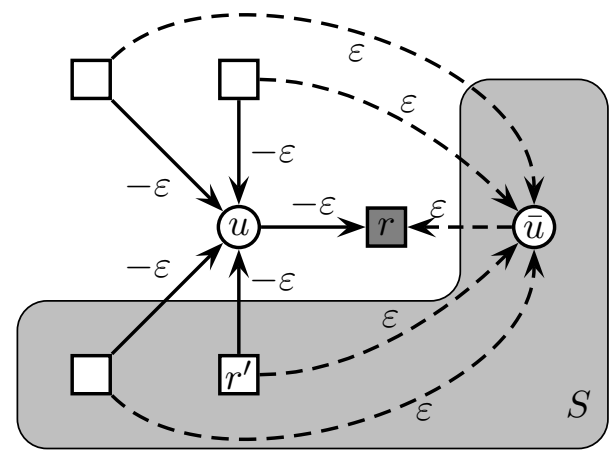

(b) capacities $\bar{x}^{(r)}$

Figure 5: Transferring capacity of $\varepsilon$ according to Lemma 4.4. $(a)$ visualizes capacity in $x^{(r)}$, where newly added edges $\bar{e}$ are dashed. (b) depicts $\bar{x}^{(r)}$ and a potentially critical cut $S$.

proof of a 1.55 upper bound. Operations Research Letters, 38(6):567 - 570, 2010.

[8] M. Chlebík and J. Chlebíková. The Steiner tree problem on graphs: Inapproximability results. Theoretical Computer Science, 406(3):207-214, 2008.

[9] E. N. Gilbert and H. O. Pollak. Steiner minimal trees. SIAM Journal on Applied Mathematics, 16(1):1-29, 1968.

[10] M. X. Goemans. Minimum bounded degree spanning trees. In Proceedings of the 47th IEEE Symposium on Foundations of Computer Science (FOCS), pages 273-282, 2006.

[11] M. X. Goemans and Y. Myung. A catalog of Steiner tree formulations. Networks, 23(1):19-28, 1993.

[12] M. Karpinski and A. Zelikovsky. New approximation algorithms for the Steiner tree problem. Journal of Combinatorial Optimization, 1(1):47-65, 1997.

[13] J. Könemann, D. Pritchard, and K. Tan. A partition-based relaxation for Steiner trees. Math. Program., 127(2):345-370, 2011.

[14] C.J.H. McDiarmid. Rado's theorem for polymatroids. Mathematical Proceedings of the Cambridge Philosophical Society, 78:263-281, 1975.

[15] N. Megiddo. Applying parallel computation algorithms in the design of serial algorithms. J. ACM, 30(4):852-865, 1983.

[16] M. Padberg and L.A. Wolsey. Trees and cuts. In Combinatorial Mathamtics (Proceedings International
Colloquium on Graph Theory and Combinatorics), pages 511-517, 1983.

[17] J.-C. Picard and M. Queyranne. Selected applications of minimum cuts in networks. INFOR Canadian Journal of Operational Research and Information Processing, 20:294-370, 1982.

[18] T. Polzin and S. Vahdati-Daneshmand. On Steiner trees and minimum spanning trees in hypergraphs. Operations Research Letters, 31(1):12-20, 2003.

[19] H. J. Prömel and A. Steger. A new approximation algorithm for the Steiner tree problem with performance ratio $5 / 3$. Journal of Algorithms, 36:89-101, 2000.

[20] S. Rajagopalan and V. V. Vazirani. On the bidirected cut relaxation for the metric Steiner tree problem. In ACM-SIAM Symposium on Discrete Algorithms (SODA), pages 742-751, 1999.

[21] G. Robins and A. Zelikovsky. Tighter bounds for graph Steiner tree approximation. SIAM Journal on Discrete Mathematics, 19(1):122-134, 2005.

[22] A. Schrijver. Combinatorial Optimization, Polyhedra and Efficiency. Springer, 2003.

[23] D. Warme. Spanning Trees in Hypergraphs with Applications to Steiner Trees. $\mathrm{PhD}$ thesis, 1998.

[24] A. Zelikovsky. An 11/6-approximation algorithm for the network Steiner problem. Algorithmica, 9:463-470, 1993. 


\section{APPENDIX}

\section{A. SEPARATION AND GAMMOID STRUC- TURE}

In this section, we investigate the separation problem for (LP). Although it is not necessary, for convenience we will work in the blown up formulation; thus, for a given $\mathcal{X}$, our goal is to determine whether (2) is satisfied (see Section 3 for details of this and the definition of $h_{\mathcal{X}}$ ). For any $Q \subseteq R, Q \neq \emptyset$, we will find the most violated set over all $S \supseteq Q$. Given this, we can answer the separation question by checking that $\min _{S \supseteq\{v\}} h_{\mathcal{X}}(S)$ is zero for each choice of $v$ (note that $h_{\mathcal{X}}(\emptyset)=-N$, and so we must exclude this trivial set from consideration). For each choice of $v$, one maxflow calculation will be required.

We note that since $h_{\mathcal{X}}$ is an intersecting submodular function, $\min _{S \supseteq Q} h_{\mathcal{X}}(S)$ can also be computed using submodular function minimization as a black box [22]. However, the combinatorial algorithm we demonstrate here gives some additional insights (as well as being more efficient).

The construction is inspired by one for the forest polytope [17, 16] (see also [22, §51.4]). While what follows is not precisely a generalization (in the case where all components have size 2 , the resulting construction is slightly different), it is similar in spirit. In the directed component-based relaxation, separation via an equivalent flow-based formulation is completely straightforward. However this does not imply such a formulation for the undirected version.

Let $\mathcal{X}$ be the blowup graph of some solution $x$. First, let

$$
y_{v}=|\{C \in \Gamma(\mathcal{X}): v \in C\}|-N \quad \text { for } v \in R .
$$

If $y_{v}$ is negative for any $v$, it is easily seen that $\mathcal{X}$ is not feasible (it corresponds to $x(\delta(v))<1$ ). So from now on, we assume $y_{v} \geq 0$ for all $v \in R$. Construct a directed multigraph $D=(W, A)$ (we will write $D_{\mathcal{X}}$ if we wish to be explicit on the choice of $\mathcal{X}$ ) as follows. Begin with the multigraph $\mathcal{X}$, and for each component $C \in \Gamma(\mathcal{X})$, pick an arbitrary vertex $r_{C}$ as the root. Adjoin a source vertex $s$ and sink vertex $t$. Now orient all edges of $E(C)$ away from $r_{C}$ for each component, and adjoin the arcs $s r_{C}$ for each $C \in \Gamma(\mathcal{X})$, and $v t$ for each $v \in R$. We assign capacities $z$ to the arcs; $z(v t)=y_{v}$ for all $v \in R$, and $z(a)=1$ for all other arcs.

THEOREM A.1. For any nonempty $Q \subseteq R$, the value of the maximum $s-(Q \cup\{t\})$-flow in $D$ is equal to

$$
y(R)+N+\min _{S \supseteq Q} h_{\mathcal{X}}(S) .
$$

Proof. Use $\vec{E}(C)$ to denote the arcs in $D$ corresponding to $E(C)$ in $\mathcal{X}$, and let $A(C)=\vec{E}(C) \cup\left\{s r_{C}\right\}$. For any $S \subseteq R$, let $\nu(S) \subseteq W$ be defined by

$$
\nu(S)=S \cup\{t\} \cup \bigcup_{\substack{C \in \Gamma(\mathcal{X}): \\ C \cap S \neq \emptyset}}(V(C) \backslash R) .
$$

Claim A.2. For any nonempty $S \subseteq R$,

$$
z\left(\delta^{-}(\nu(S))\right)=h_{\mathcal{X}}(S)+y(R)+N .
$$

Moreover, for any $U \subset W$ with $s \notin U, t \in U$ and $U \cap R=S$, we have $z\left(\delta^{-}(U)\right) \geq z\left(\delta^{-}(\nu(S))\right)$.

Proof. Consider some component $C \in \Gamma(\mathcal{X})$. If $S \cap C=\emptyset$, then clearly $\delta^{-}(\nu(S)) \cap A(C)=\emptyset$. So suppose $S \cap C \neq \emptyset$. If $r_{C} \in$ $\nu(S)$, then clearly $\delta^{-}(\nu(S)) \cap \vec{E}(C)=\emptyset$, and $s r_{C} \in \delta^{-}(\nu(S))$. On the other hand, if $r_{C} \notin \nu(S)$ (implying in particular that $r_{C}$ is a terminal), then $s r_{C} \notin \delta^{-}(\nu(S))$ and $\left|\delta^{-}(\nu(S)) \cap \vec{E}(C)\right|=1$, since all terminals are leaves of the components they belong to. In either case, $z\left(\delta^{-}(\nu(S)) \cap A(C)\right)=1$.

For any $v \in R, v t \in \delta^{-}(\nu(S))$ if and only if $v \notin S$. Putting this all together,

$$
z\left(\delta^{-}(\nu(S))\right)=|\{C \in \Gamma(\mathcal{X}): C \cap S \neq \emptyset\}|+\sum_{v \notin S} y_{v} .
$$

Now taking (1) and adding and subtracting $y(S)$, we have

$$
\begin{aligned}
h_{\mathcal{X}}(S)= & N(|S|-1)-\sum_{C \in \Gamma(\mathcal{X})}(|C \cap S|-1)^{+} \\
& +\left(\sum_{C \in \Gamma(\mathcal{X})}|C \cap S|-N|S|\right)-y(S) \\
= & |\{C \in \Gamma(\mathcal{X}): C \cap S \neq \emptyset\}|-N-y(S) \\
= & z\left(\delta^{-}(\nu(S))-N-y(R) .\right.
\end{aligned}
$$

Consider any $U$ with $t \in U, s \notin U$ and $U \cap R=S$. We again clearly have $v t \in \delta^{-}(U)$ for all $v \in R \backslash S$, and again $\delta^{-}(U) \cap$ $A(C) \neq \emptyset$ if $S \cap C \neq \emptyset$. So $z\left(\delta^{-}(U)\right) \geq z\left(\delta^{-}(\nu(S))\right)$.

Now let $U^{*}$ be any minimum $s-(Q \cup\{t\})$-cut, with $t \in U^{*}$ and $s \notin U^{*}$. By the claim, $z\left(\delta^{-}\left(\nu\left(S^{*}\right)\right) \leq z\left(\delta^{-}\left(U^{*}\right)\right)\right.$; since $U^{*}$ is a minimum cut, we must have equality. Then again by the claim,

$$
z\left(\delta^{-}\left(\nu\left(S^{*}\right)\right)\right)=h_{\mathcal{X}}\left(S^{*}\right)+y(R)+N .
$$

The result now follows by the max-flow min-cut theorem.

We now show how this leads to a description of the matroid $M_{Q}$ as a gammoid. Recall the definition of a gammoid: a directed graph $H$ is given, along with two subsets $X, Y \subseteq V(H)$. The groundset of the gammoid is $X$, and a set $I \subseteq X$ is independent if there are vertex-disjoint paths from $I$ to some subset of $Y$. We say in this case that this defines the gammoid from $X$ to $Y$ in $H$. It is convenient to observe that by transforming the digraph $H$ appropriately, we can replace vertex-disjoint in the above definition with arc-disjoint, and still characterize gammoids.

We need to slightly tweak the digraph $D$ defined above. For each $f \in E(\mathcal{X})$, there is a corresponding arc $a$ in $D$. Split the arc by adding an additional vertex $v_{f}$, producing a "front" $\operatorname{arc} a_{f}^{\mathrm{f}}$ with tail $v_{f}$ and a "back" arc $a_{f}^{\mathrm{b}}$ with head $v_{f}$. We may also remove the vertex $s$ and all its adjacent arcs. Call the resulting modified digraph $D^{\prime}$.

Define the sets

$$
X=\left\{v_{f} \mid f \in E(\mathcal{X})\right\}, \quad X^{\prime}=\bigcup_{C \in \Gamma(\mathcal{X})} r_{C} \quad \text { and } \quad Y=Q \cup\{t\} .
$$

Let $\mathfrak{G}_{Q}^{\prime}$ be the gammoid defined on $D$ from $X^{\prime} \cup X$ to $Y$, requiring arc-disjointness rather than vertex-disjointness. Then define $\mathfrak{G}_{Q}=$ $\mathfrak{G}_{Q}^{\prime} / X^{\prime}$; this contraction is also a gammoid. By the one-to-one correspondence between $X$ and $E(\mathcal{X})$, we may consider this is a matroid over $E(\mathcal{X})$.

THEOREM A.3. For any component $Q, \mathfrak{G}_{Q}=M_{Q}$.

Proof. The rank of a set $U \subseteq X$ in $\mathfrak{G}_{Q}$ is $\rho(U)=\rho^{\prime}(U \cup$ $\left.X^{\prime}\right)-\rho^{\prime}\left(X^{\prime}\right)$, where $\rho^{\prime}$ is the rank function of $\mathfrak{G}_{Q}^{\prime}$. Notice that the maximum number of arc-disjoint paths from $X^{\prime}$ to $Y$ is precisely the max-flow from $s$ to $Q \cup\{t\}$ in $D$. Thus by Theorem A.1,

$$
\begin{aligned}
\rho^{\prime}\left(X^{\prime}\right) & =y(R)+N+\min _{S \supseteq Q} h_{\mathcal{X}}(S) \\
& =y(R)+N,
\end{aligned}
$$


since by feasibility of $\mathcal{X}, h_{\mathcal{X}}(R)=0$.

Let $A_{U}:=\left\{a_{f}^{\mathrm{b}} \mid v_{f} \in U\right\}$, and let $D_{U}^{\prime}$ be the subgraph of $D^{\prime}$ obtained by removing $A_{U}$. Now $\rho^{\prime}\left(U \cup X^{\prime}\right)$ is the maximum number of arc-disjoint paths from $U \cup X^{\prime}$ to $Y$ in $D^{\prime}$; we claim it is also the number of such paths in $D_{U}^{\prime}$. For take $P_{1}, \ldots, P_{\ell}$ to be any maximum collection of arc-disjoint paths from $U \cup X^{\prime}$ to $Y$ in $D^{\prime}$. For some $v_{f} \in U$, if some path $P_{i}$ uses arc $a_{f}^{\mathrm{b}}$, then certainly no other path emanates from $v_{f}$, and so we can simply remove the initial segment of $P_{i}$ before $v_{f}$ to obtain another maximum collection of disjoint paths that do not use $a_{f}^{\mathrm{b}}$. Repeating this process, we obtain paths $P_{1}^{\prime}, \ldots, P_{\ell}^{\prime}$ that do not use any arcs in $A_{U}$, and so give a feasible collection of arc-disjoint paths in $D_{U}^{\prime}$.

But by contracting all of $U$ to form a source, $D_{U}^{\prime}$ corresponds exactly to $D_{\mathcal{X}-U}$, the digraph for the separation construction corresponding to $\mathcal{X}-U$. So the maximum $s-(Q \cup\{t\})$-flow in $D_{\mathcal{X}-U}$ has value $\rho^{\prime}\left(U \cup X^{\prime}\right)$. Applying Theorem A.1,

$$
\begin{aligned}
\rho^{\prime}\left(U \cup X^{\prime}\right) & =y(R)+N+\min _{S \supseteq Q} h_{\mathcal{X}-U}(S) \\
& =\rho^{\prime}\left(X^{\prime}\right)+r_{Q}(U),
\end{aligned}
$$

by (10) as well as the definition of $r_{Q}$. Thus $\rho(U)=r_{Q}(U)$, and so $\mathfrak{G}_{Q}=M_{Q}$.

\section{B. PROOFS FOR SECTION 2.3}

Let us fix a component $C \in \Gamma(\mathcal{X})$ and a splitting set $K$. By the definition of $K$ (as the complement of a spanning tree in the graph $\tilde{C}$ obtained by contracting terminals), every Steiner vertex $u \in V(C) \backslash C$ has a unique path $P_{u} \subseteq E(C) \backslash K$ of cleanup edges to a terminal that we term $r_{u} \in C$ (see again Figure 3).

LEMMA B.1. For any splitting set $K \subseteq E(\mathcal{X})$, component $C \in \Gamma(\mathcal{X})$ and edge $e \in E(C) \backslash K$, let

$$
W(e)=\left\{u v \in K \cap E(C) \mid e \in P_{u}\right\} .
$$

Then $W(e)$ is the unique minimal subset of $E(C) \cap K$ whose removal makes e a pendant edge.

Proof. Let $\bar{W} \subseteq E(C) \cap K$ be any subset of splitting edges. If $u v \in W(e) \backslash \bar{W}$ then $e$ remains on a path, namely $P_{u} \cup u v \cup P_{v} \subseteq$ $E(C) \backslash \bar{W}$ between the terminals, implying that $e$ is not pendant. Thus, any subset $\bar{W}$ which makes $e$ pendant must contain $W(e)$.

On the other hand, we claim that $e$ is pendant in $E(C) \backslash W(e)$. To see this, let $e=u v$ with $e \in P_{u}$. For $e$ not to be pendant, there would need to be a path $P \subseteq E(C) \backslash(W(e) \cup\{e\})$ from $u$ to a terminal. But the first edge in $K$ on $P$ must be in $W(e)$, contradicting the fact that $P \cap W(e)=\emptyset$.

THEOREM 2.4 1. For any splitting set $K$ and feasible blowup graph $\mathcal{X}$, Algorithm 1 yields a solution of cost at most $\Phi_{K}(\mathcal{X}) / N$.

PROOF. We prove the theorem by showing that the decrease in the potential at any iteration is lower bounded by the weight of the edges we remove. More formally, consider a given iteration $t$ with current blowup graph $\mathcal{X}_{t}$, splitting set $K_{t}$, and weights $w_{t}$. let $Q_{t}$ be the component to contract and $B_{t} \in \mathcal{B}_{Q}^{K_{t}}$ the edges to be removed from $\mathcal{X}_{t}$ in this iteration. At the end of iteration $t$ a new blowup graph $\mathcal{X}_{t+1}$ is obtained with splitting set $K_{t+1}=K_{t} \backslash B_{t}$. We will show

$$
\Phi_{K_{t}}\left(\mathcal{X}_{t}\right)-\Phi_{K_{t+1}}\left(\mathcal{X}_{t+1}\right) \geq w_{t}\left(B_{t}\right) .
$$

This in turn implies the theorem since the potential function at any iteration, and in particular at the end of the algorithm, is nonnegative. Therefore, the total weight of all core edges being removed throughout the algorithm is upper bounded by the potential value of the initial blowup graph, i.e., $\sum_{t} w_{t}\left(B_{t}\right) \leq \Phi_{K}(\mathcal{X})$. Furthermore, since at every iteration, $Q_{t}$ and $B_{t}$ are chosen such that $\operatorname{cost}\left(Q_{t}\right) \leq$ $w_{t}\left(B_{t}\right) / N$, we obtain that the cost of all contracted componentswhich is the cost of the Steiner tree our algorithm returns-can be upper bounded by $\sum_{t} \operatorname{cost}\left(Q_{t}\right) \leq \frac{1}{N} \sum_{t} w_{t}\left(B_{t}\right) \leq \Phi_{K}(\mathcal{X}) / N$, as desired. Hence, it remains to prove (11).

For any edge $e \in \mathcal{X}_{t}$, we denote by $W_{t}(e)$ its witness set at the beginning of iteration $t$. For simplicity, we define $W_{t+1}$ on all of $E\left(\mathcal{X}_{t}\right)$, defining $W_{t+1}(e)=\emptyset$ for $e \in E\left(\mathcal{X}_{t}\right) \backslash E\left(\mathcal{X}_{t+1}\right)$. By definition of the witness sets, we have

$$
W_{t+1}(e)=W_{t}(e) \backslash B_{t} \quad \text { for any } e \in E\left(\mathcal{X}_{t}\right) .
$$

Expanding the left-hand side of (11), we obtain

$$
\begin{aligned}
& \sum_{K_{t}}\left(\mathcal{X}_{t}\right)-\Phi_{K_{t+1}}\left(\mathcal{X}_{t+1}\right) \\
= & \sum_{e \in E\left(\mathcal{X}_{t}\right)} c(e)\left(H\left(\left|W_{t}(e)\right|\right)-H\left(\left|W_{t+1}(e)\right|\right)\right) \\
\geq & \sum_{e \in E\left(\mathcal{X}_{t}\right)} c(e) \sum_{k=\left|W_{t+1}(e)\right|+1} \frac{\left|W_{t}(e)\right|}{k} \cdot \frac{\left|W_{t}(e)\right|-\left|W_{t+1}(e)\right|}{\left|W_{t}(e)\right|} \\
= & \sum_{e \in E\left(\mathcal{X}_{t}\right)} c(e) \cdot \frac{\left|W_{t}(e) \cap B_{t}\right|}{\left|W_{t}(e)\right|} \text { by (12). }
\end{aligned}
$$

Furthermore, by expanding the right-hand side of (11) using the definition of the weights $w_{t}$, we obtain

$$
\begin{aligned}
w_{t}\left(B_{t}\right) & =\sum_{f \in B_{t}} \sum_{\substack{e \in E\left(\mathcal{X}_{t}\right) \\
e \in W_{t}(f)}} \frac{c(e)}{\left|W_{t}(e)\right|} \\
& =\sum_{e \in E\left(\mathcal{X}_{t}\right)} \sum_{f \in B_{t} \cap W_{t}(e)} \frac{c(e)}{\left|W_{t}(e)\right|} \\
& =\sum_{e \in E\left(\mathcal{X}_{t}\right)} c(e) \frac{\left|W_{t}(e) \cap B_{t}\right|}{\left|W_{t}(e)\right|} .
\end{aligned}
$$

Inequality (11) finally follows by combining (13) with (14).

In the following, we show that $K$ can always be chosen s.t. $\Phi_{K}(\mathcal{X}) \leq \ln (4) \cdot \operatorname{cost}(\mathcal{X})$, following the proof of [4]. For the sake of a simpler exposition, we replace every Steiner vertex in $\mathcal{X}$ of degree higher than 3 , with a binary tree consisting of cost zero edges in order to obtain vertices that have degree exactly 3 . Suppose we find a suitable pair $(K, F)$ of splitting and cleanup edges in this auxiliary graph. Then every Steiner vertex $u$ in the original graph has potentially several paths $P_{1}, \ldots, P_{q} \subseteq F$ of cleanup edges to terminals. We keep the one path minimizing $c\left(P_{i}\right)$ and discard the first edge of all other paths. This does not increase $\Phi_{K}(\mathcal{X})$. Applying this iteratively, we end up with a feasible pair of cleanup edges and splitting edges.

From now on, we assume that every component $C \in \Gamma(\mathcal{X})$ is a binary tree. We pick an arbitrary edge $e_{C} \in E(C)$ as root edge. From any interior vertex $u \in V(C) \backslash C$, there are two outgoing edges (these are the edges that do not lie on the path from $u$ to the root edge). We randomly pick one of these edges as cleanup edge and the other one as splitting edge. In other words, every interior vertex $u$ has a unique path of cleanup edges to some terminal and hence, $K$ is a legal splitting set. Moreover, for every non-root edge $e$ one has $\mathbb{P}\{e \in K\}=\frac{1}{2}$. 
LEMMA B.2. If $E(\mathcal{X})$ is chosen randomly according to the above distribution,

$$
\mathbb{E}\left\{\Phi_{K}(\mathcal{X})\right\} \leq \ln (4) \cdot \operatorname{cost}(\mathcal{X}) .
$$

Proof. Fix a component $C$ and an edge $e \in E(C)$. It suffices to show that $\mathbb{E}\{H(|W(e)|)\} \leq \ln (4)$. The root edge is always a splitting edge, thus $\left|W\left(e_{C}\right)\right|=1$. So, let $e$ be a non-root edge and let $v_{0}, v_{1}, \ldots, v_{k+1}$ be the path from $e$ to the root edge, i.e. $v_{0} v_{1}=e$ and $v_{k} v_{k+1}=e_{C}$. Let

$$
X:=\max \left\{i \mid v_{0} v_{1}, v_{1} v_{2}, \ldots, v_{i-1} v_{i} \in E(C) \backslash K\right\}
$$

be the number of consecutive cleanup edges on this path, starting from $e$ (and $X=0$ if already $\left.v_{0} v_{1} \in K\right)$. Then $\mathbb{P}\{X=i\}=$ $\left(\frac{1}{2}\right)^{i+1}$ for $i<k$ and $\mathbb{P}\{X=k\}=\left(\frac{1}{2}\right)^{k}$. Furthermore $|W(e)|=$ $X+1$ if $X<k$ and $|W(e)|=k$ otherwise. We calculate

$$
\begin{aligned}
\mathbb{E}\{H(|W(e)|)\} & \leq \sum_{i=0}^{k-1} \mathbb{P}\{X=i\} \cdot H(i+1)+\mathbb{P}\{X=k\} \cdot H(k) \\
& \leq \sum_{i=0}^{\infty} H(i+1) \cdot\left(\frac{1}{2}\right)^{i+1} \\
& =\ln (4) .
\end{aligned}
$$

The above argument can be derandomized by the method of conditional expectations, and this leads to a proof of Theorem 2.5. Another option is to observe that the best choice of $K$ can be found in polynomial time, via a dynamic program as is indicated below. Combined with the above lemma, this implies Theorem 2.5.

Lemma B.3. A splitting set $K$ minimizing $\Phi_{K}(\mathcal{X})$ can be found in polynomial time.

PROOF. Since the potential function can be decomposed into terms corresponding to each component, and a splitting set $K$ consists of the union of splitting sets in each component, it suffices to consider each component separately. Hence, let $C$ be any fixed component with vertices $V(C)$ and edges $E(C)$; our goal is to find a splitting set $K$ for $C$ that minimizes $\sum_{e \in E(C)} c(e) H(|W(e)|)$.

As usual when applying dynamic programming to problems on trees, we start by computing tables (to be specified soon) for subtrees consisting of a single terminal, and successively combine those tables until a table for the full tree is obtained, revealing the optimal splitting set. To specify the order in which we create tables for larger subtrees from smaller ones, we direct the edge of the tree $C$ away from an arbitrarily chosen vertex in $V(C)$. We consider the following type of subtrees that we call partial trees. For any vertex $r \in V(C)$ and subset $U \subseteq \delta^{+}(r)$ of arcs leaving $r$, the partial tree $T_{U}$ with root $r$ is the induced subgraph of $C$ consisting of $r$ and all vertices that can be reached from $r$ with paths starting with one of the $\operatorname{arcs}$ in $U$. To simplify notation we also use $T_{U}$ to refer to the edge set of the partial tree. Furthermore, let $\bar{T}_{U}=E(C) \backslash T_{U}$, and let $R_{T_{U}} \subseteq R$ denote the terminals contained in the partial tree $T_{U}$.

To better understand what information should be stored for a partial tree $T$, we first briefly discuss how the choice of splitting set $K$ within $T$ impacts the witness sets in $\bar{T}$, and vice versa. We will refer to the choice of core and cleanup edges (i.e., the choice of $K$ ) within some subset of edges as a configuration for that subset. We distinguish two ways that the root $r$ of $T$ can be connected to a terminal through cleanup edges: case $(A)$ through a path within the partial tree $T$, and case $(B)$ through a path outside of $T$. Correspondingly, we call a configuration for $T$ a type $(A)$ configuration if case (A) holds for the root of $T$, and a type (B) configuration otherwise.
Notice that in a type (A) configuration, every vertex within $T$ is connected to a terminal in $R_{T}$ by cleanup edges. For a partial tree $T$ we will store two tables, one corresponding to case (A) and one to case (B).

Consider case (A) and let $P$ be the path of cleanup edges connecting a terminal in $R_{T}$ to $r$. Notice that in this case $W(e) \subseteq \bar{T} \forall e \in$ $\bar{T}$. Hence, the configuration for $T$ does not have any impact on the contribution of the edges of $\bar{T}$ to the function $\sum_{e \in E(C)} c(e) H(|W(e)|)$. However, the witness sets of the edges in $P$ depend on the configuration for $\bar{T}$, namely every core edge that can be reached within $\bar{T}$ from $r$ by following cleanup edges is part of the witness set of any $e \in P$. Hence, the only information about the configuration for $\bar{T}$ that matters in finding an optimal configuration within $T$ is the number $\alpha$ of core edges in $\bar{T}$ that can be reached from $r$ through cleanup edges. Thus for case (A) we want to store a table for $T$ which contains, for each value of $\alpha \in\{0, \ldots,|\bar{T}|\}$, a corresponding type (A) configuration that minimizes $\sum_{e \in T} c_{e} H(|W(e)|)$. Here, $|W(e)|$ can be computed without knowing the precise configuration in $\bar{T}$ (apart from $\alpha$ ) since

$$
|W(e)|= \begin{cases}|W(e) \cap T| & \text { if } e \in T \backslash P \\ |W(e) \cap T|+\alpha & \text { if } e \in P\end{cases}
$$

Now consider case (B) and let $P$ be the path in $\bar{T}$ connecting a terminal to $r$. In this case the situation is reversed and $W(e) \subseteq T$ for any $e \in T$. Hence, the configuration for $\bar{T}$ does not have any impact on the contribution of the edges of $T$ to the function $\sum_{e \in E(C)} c(e) H(|W(e)|)$. However this time, the witness sets of edges on $P$ depends on the configuration for $T$, namely every core edges that can be reached within $T$ from $r$ by following cleanup edges is part of the witness set of any $e \in P$. Hence, the only information that has to be stored for $T$ in case (B), in order to describe how the configuration within $T$ impacts the configuration outside of $T$, is the number $\beta$ of core edges in $T$ that can be reached from $r$ through cleanup edges. Hence, for case (B) we want to store a table for $T$ which contains, for each value of $\beta \in\{0, \ldots,|T|\}$, a corresponding type (B) configuration that minimizes $\sum_{e \in T} c(e) H(|W(e)|)$.

Clearly, if we can compute the (A) table for the full component $C$, then we are done, since the globally best configuration is the one minimizing the potential function over all values of $\alpha$. Computing type (A) and (B) tables for partial trees corresponding to single terminals is trivial: table (A) contains one entry corresponding to $\alpha=0$ of value zero, and table (B) is empty. There are two constellation we exploit to compute tables for larger partial trees based on the tables of smaller ones.

The first constellation is the following. Assume that we have tables (A) and (B) for two partial trees $T_{U_{1}}$ and $T_{U_{2}}$ with $U_{1} \cap U_{2}=\emptyset$, and both having root $r$. Then we can compute the two tables for $T_{U_{1} \cup U_{2}}$ from the tables of $T_{U_{1}}$ and $T_{U_{2}}$. This can be done by considering all legal combinations (meaning pairs of configurations that can be completed to a splitting set) of one table entry corresponding to $T_{U_{1}}$ and one corresponding to $T_{U_{2}}$, keeping the best ones. Since the size of each table is polynomially bounded in the input, this can be done efficiently. We skip the somewhat tedious details for combining those tables which are based on standard arguments.

In the second constellation, we consider a vertex $r$ and one of its out-neighbors $v$, i.e., there is an arc directed from $r$ to $v$, such that both tables for $T_{\delta}+(v)$ have already been computed. We can then compute the two tables for $T_{\{r v\}}$ by considering all legal combinations of an entry of one of the tables of $T_{\delta}+(v)$ and the two possibilities of $r v$ being a core edge or a cleanup edge.

It is easy to observe that starting from the terminals and leveraging 
the above two update rules, one can construct both tables for the full component $C$ efficiently.

For the following Lemma, we assume that the graph $G$ is quasibipartite.

LEMMA 4.1 1. Let $K=E(\mathcal{X}) \backslash E_{\text {min }}$, where $E_{\text {min }}$ consists of a cheapest edge from every component. Then

$$
\Phi_{K}(\mathcal{X}) \leq \frac{73}{60} \cdot \operatorname{cost}(\mathcal{X}) .
$$

ProOF. Consider a component $C$, which now is a star with edges $e_{1}, \ldots, e_{k}$. Assume $e_{k}$ minimizes the cost, then the splitting edges in $C$ are $K \cap C=\left\{e_{1}, \ldots, e_{k-1}\right\}$. First of all, $K$ is obviously a legal splitting set. Secondly $\left|W\left(e_{i}\right)\right|=1$ for $i \in\{1, \ldots, k-1\}$ and $\left|W\left(e_{k}\right)\right|=k-1$. Thus

$\sum_{i=1}^{k} c(e) \cdot H\left(\left|W\left(e_{i}\right)\right|\right) \leq(k-1+H(k-1)) \cdot \frac{\operatorname{cost}(C)}{k} \leq \frac{73}{60} \cdot \operatorname{cost}(C)$,

using that $1+\frac{H(k-1)-1}{k}$ is maximized for $k=5$. The claim follows by summing over all components $C \in \Gamma(\mathcal{X})$.

\section{A LOWER-BOUND PROPERTY OF NONNEGATIVE INTERSECTING SUBMODULAR FUNCTIONS}

The main goal of this section is to prove Theorem 3.6. Before presenting the core part of the proof we discuss some basic properties of partition functions, and make some general observations concerning the statement of Theorem 3.6 which are useful to understanding its proof.

Let $U$ be a finite set. We recall that $\mathcal{F} \subseteq 2^{U}$ is called a lattice family if it is closed under unions and intersections. A function $\mathcal{F} \rightarrow$ $\mathbb{R}$ is submodular on $\mathcal{F}$ if $f(A \cup B)+f(A \cap B) \leq f(A)+f(B)$ for all $A, B \in \mathcal{F}$; supermodular on $\mathcal{F}$, intersecting supermodular on $\mathcal{F}$ etc., are defined similarly in the obvious way.

Any partition $\mathcal{P}=\left\{P_{1}, \ldots, P_{n}\right\}$ of $U$ induces naturally a lattice family $\mathcal{F}_{\mathcal{P}} \subseteq 2^{U}$ which consists of all possible unions of sets in $\mathcal{P}$. Consider the coverage function $\alpha(S)=\mid\left\{j \in[n] \mid P_{j} \cap\right.$ $S \neq \emptyset\} \mid$, which is clearly submodular. Notice that we can write $f_{\mathcal{P}}(S)=(\alpha(S)-1)^{+}$, and in particular $f_{\mathcal{P}}(S)=\alpha(S)-1$ for all $S \neq \emptyset$. Thus $f_{\mathcal{P}}$ is intersecting submodular: for any $A, B \subseteq U$ with $A \cap B \neq \emptyset$,

$$
\begin{aligned}
f_{\mathcal{P}}(A)+f_{\mathcal{P}}(B) & =(\alpha(A)-1)+(\alpha(B)-1) \\
& \geq \alpha(A \cup B)-1+\alpha(A \cap B)-1 \\
& =f_{\mathcal{P}}(A \cup B)+f_{\mathcal{P}}(A \cap B) .
\end{aligned}
$$

Furthermore, it is easy to see that $f_{\mathcal{P}}$ is intersecting supermodular on $\mathcal{F}_{\mathcal{P}}$. Hence $f_{\mathcal{P}}$ is intersecting modular on $\mathcal{F}_{\mathcal{P}}$, i.e., $f_{\mathcal{P}}(A)+$ $f_{\mathcal{P}}(B)=f_{\mathcal{P}}(A \cup B)+f_{\mathcal{P}}(A \cap B)$ for any intersecting sets $A, B \in \mathcal{F}_{\mathcal{P}}$.

By the above observation, the function $f$ claimed by Theorem 3.6 is by construction intersecting submodular since all $f_{\mathcal{P}^{i}}$ are intersecting submodular. Similarly, $f$ is monotone due to the monotonicity of $f_{\mathcal{P}^{i}}$. We prove the following slightly stronger version of Theorem 3.6.

THEOREM C.1. Let $h: 2^{U} \rightarrow \mathbb{R}_{+}$any nonnegative intersecting submodular function, such that all maximal sets $S \subseteq U$ with $h(S)=0$ form a partition $\mathcal{P}^{1}$ of $U$. Then there is an intersecting submodular function $f$ of the form

$$
f=\sum_{i=1}^{k} \lambda_{i} f_{\mathcal{P}^{i}}
$$

where $k \leq|U|-1, \lambda_{i}>0 \forall i \in[k], \mathcal{P}^{1}, \ldots \mathcal{P}^{k}$ are partitions of $U$ that become coarser with increasing index, and $f$ satisfies:

i) $f(S) \leq h(S) \quad \forall S \subseteq U$,

ii) $f(U)=h(U)$.

Furthermore, the partitions $\mathcal{P}^{i}$ together with the coefficients $\lambda_{i}$, and hence $f$, can be constructed efficiently.

Notice that the condition in Theorem C.1 stating that the maximal tight sets of $h$ form a partition of $U$ is equivalent to the property that the family of all tight sets of $h$ covers $U$, due to the following uncrossing argument. If the tight sets of $h$ cover $U$ then so do the maximal tight sets; furthermore, for any two intersecting tight sets $A, B \subseteq U$

$$
0=h(A)+h(B) \geq h(A \cup B)+h(A \cap B) \geq 0,
$$

by intersecting submodularity and nonnegativity of $h$; hence $A \cup B$ is also tight. Hence, this condition is indeed weaker than the one used in Theorem 3.6, which states that all singletons must be tight.

Proof of Theorem C. 1 . The partitions $\mathcal{P}^{1}, \ldots, \mathcal{P}^{k}$ and coefficients $\lambda_{1}, \ldots, \lambda_{k}$ defining $f$ are obtained as follows.

1. Let $i=1, h^{1}=h$, and $\mathcal{P}^{1}$ be the maximal tight sets with respect to $h$.

2. While $h^{i}(U)>0$ :

(a) Let $\lambda_{i} \in \mathbb{R}_{+}$be the maximum value such that

$$
h^{i}(S)-\lambda_{i} f_{\mathcal{P}^{i}}(S) \geq 0 \quad \forall S \in \mathcal{F}_{\mathcal{P}^{i}} .
$$

(b) $h^{i+1} \leftarrow h^{i}-\lambda_{i} f_{\mathcal{P}^{i}}$; let $\mathcal{P}^{i+1} \subseteq \mathcal{F}_{\mathcal{P}^{i}}$ be the maximal tight sets with respect to $h^{i+1}$.

(c) $i \leftarrow i+1$.

We start by observing that each function $h^{i}$ encountered during the algorithm is intersecting submodular over $\mathcal{F}_{\mathcal{P}^{i-1}}$ (by convention we set $\mathcal{P}^{0}=2^{U}$ ), and that $\mathcal{P}^{i}$ indeed forms a partition of $U$. This can easily be verified through an inductive argument. By assumption $h^{1}$ is intersecting submodular over $U$, and $\mathcal{P}^{0}$ is a partition of $U$. The intersecting submodularity of $h^{i+1}=h^{i}-\lambda_{i} f_{\mathcal{P}^{i}}$ over $\mathcal{F}_{\mathcal{P}^{i}}$ follows by the intersecting submodularity of $h^{i}$ over $\mathcal{F}_{\mathcal{P}^{i}}$ and the intersecting (super)modularity of $f_{\mathcal{P}^{i}}$ over $\mathcal{F}_{\mathcal{P}^{i}}$. Since $h^{i+1}$ is intersecting submodular over $\mathcal{F}_{\mathcal{P}^{i}}$, the maximal tight sets $\mathcal{P}^{i+1}$ of $h^{i+1}$ in $\mathcal{F}_{\mathcal{P}^{i}}$ thus again form a partition of $U$.

The suggested procedure can indeed be implemented efficiently. At any iteration $i$ and for any fixed $\lambda>0$, finding the set $S \in \mathcal{F}_{\mathcal{P}^{i}}$ minimizing $h^{i}(S)-\lambda f_{\mathcal{P}^{i}}$ is an intersecting submodular function minimization problem. Hence, in step ( $2 a), \lambda_{i}$ can be found by using e.g. binary search, or by applying the parametric search technique of Megiddo [15].

Furthermore, since $f_{\mathcal{P}_{i}}(S)=0$ for all the sets $S \in \mathcal{F}_{\mathcal{P}^{i}}$ that are tight with respect to $h^{i}$-which are precisely the sets in $\mathcal{P}^{i}$-we have $\lambda_{i}>0$ in each iteration. By choosing $\lambda_{i}$ to be maximum in step (2a), there is at least one set $S \in \mathcal{F}_{\mathcal{P}^{i}}$ that is tight with respect to $h^{i+1}$ but not $h^{i}$. Hence, $\left|\mathcal{P}^{1}\right|>\left|\mathcal{P}^{2}\right|>\ldots$, and the procedures will terminate. Let $k$ be the index of the last $\lambda$ that was set in step (2a). Hence, $h^{k+1}(U)=0$, and $\mathcal{P}^{k+1}=\{U\}$. Since we start with $\left|\mathcal{P}^{0}\right| \leq|U|$ and the partitions coarsen at each step, this implies $k \leq|U|-1$. Additionally, point ii) of Theorem C.1 clearly holds by the termination criterion of the while-loop.

Hence, it remains to prove point i), which we prove by showing the following claim through induction from $j=k+1$ to $j=1$, where $j=1$ corresponds to the statement i):

$$
h^{j}(S)-\sum_{i=j}^{k} \lambda_{i} f_{\mathcal{P}^{i}}(S) \geq 0 \quad \forall S \in \mathcal{F}_{\mathcal{P}^{j-1}} .
$$


For $j=k+1$, (15) clearly holds, since $h^{k+1}(S)=h^{k}(S)-$ $\lambda_{k} f_{\mathcal{P}^{k}}(S) \geq 0 \forall S \in \mathcal{F}_{\mathcal{P}^{k}}$, by choice of $\lambda_{k}$. Now let $j \in$ $\{1, \ldots, k\}$ and assume that (15) holds for all values above $j$. Let $S \in \mathcal{F}_{\mathcal{P}^{j-1}}$, and we define $S^{\prime} \in \mathcal{F}_{\mathcal{P}^{j}}$ to be the minimal set in $\mathcal{F}_{\mathcal{P}^{j}}$ that contains $S$, i.e.,

$$
S^{\prime}:=\bigcup_{\substack{P \in \mathcal{P}^{j} \\ P \cap S \neq \emptyset}} P
$$

Notice that

$$
h^{j}(S)=h^{j}(S)+\sum_{\substack{P \in \mathcal{P}^{j} \\ P \cap S \neq \emptyset}} h^{j}(P) \geq h^{j}\left(S^{\prime}\right),
$$

where the equality holds since all sets in $\mathcal{P}^{j}$ are tight with respect to $h^{j}$ by construction, and the inequality follows by standard uncrossing arguments: for any set $T \subseteq U$ and any $P \in \mathcal{P}^{j}$ with $P \cap T \neq \emptyset$, we have $h^{j}(T)+h^{j}(P) \geq h^{j}(T \cup P)$ by intersecting submodularity and nonnegativity of $h^{j}$. Thus starting with $T=S$ and any $P \in \mathcal{P}^{j}, P \cap S \neq \emptyset$, the two terms $h^{j}(S)$ and $h^{j}(P)$ in (16) can be replaced by $h^{j}(S \cup P)$, and this procedure can be repeated until one reaches $h^{j}\left(S^{\prime}\right)$. In other words, we simply exploit that any nonnegative intersecting submodular function has the subadditivity property for any family of sets that are connected when seen as hyperedges on the given ground set.

The inductive step of the proof of (15) finally follows by

$$
\begin{aligned}
h^{j}(S)-\sum_{i=j}^{k} \lambda_{i} f_{\mathcal{P}^{i}}(S) & \geq h^{j}\left(S^{\prime}\right)-\sum_{i=j}^{k} \lambda_{i} f_{\mathcal{P}^{i}}\left(S^{\prime}\right) \\
& =h^{j+1}\left(S^{\prime}\right)-\sum_{i=j+1}^{k} \lambda_{i} f_{\mathcal{P}^{i}}\left(S^{\prime}\right) \geq 0,
\end{aligned}
$$

where the first inequality follows from (16) and the monotonicity of $\sum_{i=1}^{k} \lambda_{i} f_{\mathcal{P}^{i}}(S)$, and the last one by the inductive hypothesis.

\section{NP-HARDNESS FOR SOLVING THE COMPONENT-BASED RELAXATION}

It is well-known that there is a PTAS for solving (LP). In other words, for every fixed $\varepsilon>0$, there is a polynomial time algorithm that computes a feasible fractional solution to the considered hypergraphic relaxation (LP), which is within a $1+\varepsilon$ factor of the optimum fractional value. We argue now, that this is best possible (answering the question posed in [6]).
THEOREM D.1. Solving (LP) is strongly NP-hard.

Proof. Let $G=(V, E)$ be a complete graph with terminals $R=\left\{s_{1}, \ldots, s_{k}\right\} \subseteq V$, edge cost $c(e) \in\{1,2\}$ for all $e \in$ $E$. Bern and Plassmann [1] showed that it is NP-hard to decide whether the cost $O P T$ of the cheapest Steiner tree is at most a given parameter $Z$.

We construct another Steiner tree instance $G^{\prime}=\left(V^{\prime}, E^{\prime}\right)$ as follows: For each terminal $s_{i} \in R$ in the original instance, we add a terminal $s_{i}^{\prime}$ and an edge $s_{i} s_{i}^{\prime}$ with $\operatorname{cost} c\left(s_{i}, s_{i}^{\prime}\right):=M$ with $M:=$ $3 n^{2}$ and $n=|V|$. Furthermore, we downgrade the original terminal to an ordinary vertex, i.e., we define $R^{\prime}:=\left\{s_{i}^{\prime} \mid i=1, \ldots, k\right\}$ as terminal set. Let $O P T_{f}^{\prime}$ be the value of the optimum fractional solution of (LP) for instance $G^{\prime}$ (using components of arbitrary size).

First we show that $O P T \leq Z \Rightarrow O P T_{f}^{\prime} \leq Z+k \cdot M$. Let $S^{*}$ be the optimum integral Steiner tree in $G$. We add all $s_{i} s_{i}^{\prime}$ edges to $S^{*}$ and consider the emerging tree as component with fractional weight 1 and cost $O P T+k \cdot M$.

Next, we prove that $O P T \geq Z+1 \Rightarrow O P T_{f}^{\prime} \geq Z+1+k \cdot M$ (which in turn implies the claim of the theorem). Let $x$ be an optimum solution to (LP) in $G^{\prime}$. For a component $C \in \mathcal{K}$, we denote $E(C)$ as the contained edges from the original graph (i.e. without $s_{i} s_{i}^{\prime}$ edges) and by $|C|$ we denote the number of terminals. Either $C$ contains less than $k$ terminals, or $\operatorname{cost}(E(C)) \geq Z+1$. In any case

$$
\begin{aligned}
\frac{\operatorname{cost}(E(C))+M}{|C|-1} & \geq \min \left\{\frac{Z+1+M}{k-1}, \frac{M}{k-2}\right\} \\
& \geq \frac{Z+1+M}{k-1},
\end{aligned}
$$

using that $M=3 n^{2}, k \leq n$ and $Z \leq 2 n$. Now we can bound the cost of the fractional solution as

$$
\begin{aligned}
O P T_{f}^{\prime} & =\sum_{C \in \mathcal{K}}(\operatorname{cost}(E(C))+|C| \cdot M) \cdot x_{C} \\
& =M \sum_{C \in \mathcal{K}}(|C|-1) x_{C}+\sum_{C \in \mathcal{K}}(\operatorname{cost}(E(C))+M) x_{C} \\
& \geq(k-1) M+\sum_{C \in \mathcal{K}} \frac{|C|-1}{k-1}(Z+1+M) x_{C} \\
& =Z+1+k M
\end{aligned}
$$

exploiting $\sum_{C \in \mathcal{K}} x_{C}(|C|-1)=k-1$.

Observe that the above reduction in not approximation preserving. This is not surprising, considering the fact that Steiner tree is APX-hard even with edge weights $\{1,2\}$ (e.g., by a straightforward reduction from Set Cover with sets of size 3 ). 